\title{
The Influence of Solar System Oscillation on the Variability of the Total Solar Irradiance
}

\author{
Harald Yndestad* \\ Norwegian University of Science and Technology Aalesund, 6025 Aalesund, Norway \\ Jan-Erik Solheim ${ }^{1, *}$ \\ Department of Physics and Technology UiT The Arctic University of Norway, 9037 \\ Tromsø, Norway
}

\begin{abstract}
The total solar irradiation (TSI) is the primary quantity of energy that is provided to the Earth. The properties of the TSI variability are critical for understanding the cause of the irradiation variability and its expected influence on climate variations. A deterministic property of TSI variability can provide information about future irradiation variability and expected long-term climate variation, whereas the non-deterministic variability can only explain the past. This study of solar variability is based on an analysis of the TSI data series from 1700 A.D. and 1000 A.D., a sunspot data series from 1611 A.D., and a solar orbit data series from 1000 A.D. The study is based on a wavelet spectrum analysis. First the TSI data series are transformed into a wavelet spectrum. Then the wavelet spectrum is transformed into an autocorrelation spectrum, to identify stationary, subharmonic and coincidence periods in the TSI variability.

The results indicate that the TSI and sunspot data series have periodic cycles that is correlated to the solar position oscillation and controlled by gravity force variations from the large planets Jupiter, Saturn, Uranus and Neptune and the solar dynamo. A possible explanation is forced oscillations between the large
\end{abstract}

\footnotetext{
* Corresponding author

Email address: Harald.Yndestad@ntnu.no (Harald Yndestad)

${ }^{1}$ Retired, Address now: Wilh. Wilhemsen v 71, 1362 Hosle, Norway
} 
planets and the solar dynamo.The major solar variability is controlled by the 12-year Jupiter period and the 84-year Uranus period. The TSI data series from 1700 A. D.has a direct relation to the 84-year Uranus period with subharmonics. The phase lag between the solar position oscillation and this TSI oscillation is estimated to about $0.15 \pi$ (rad/year) for the dominating 84-year period, and is phase locked to the perihelion state of Uranus.

The long TSI data series from 1000 A.D. has stationary periods of approximately 125 years and 210 years, which are controlled by the same stationary period of 84 year. The minimum of the 125 year period coincide with the Uranus perihelion. The 125-year and the 210-year period subsequently produce a new set of subharmonic periods. The sunspot data series from 1610 A.D. has a stationary 12-year Jupiter period and a stationary period of approximately 210 years, which is controlled by a $5 / 2$ resonance to the 84 -year Uranus period. The study confirms that the 12-year Jupiter period and the 210-year de Vries/Suess period have coincidence periods in TSI and sunspot variability. The phase lag between the solar position oscillation and TSI and sunspot oscillation is estimated to about $0.7 \pi$ ( $\mathrm{rad} /$ year) for the dominating 210-year period.

A model of the stationary periods in TSI and sunspot variability confirms the results by a close relation to known long solar minimum periods since 1000 A.D. and a modern maximum period from 1940 to 2015. The model computes a new Dalton sunspot minimum from approximately 2025 to 2050 and a new Dalton period TSI minimum from approximately 2040 to 2065.

Keywords:

solar oscillation, solar irradiation oscillation, wavelet analysis, grand minima

\section{Introduction}

The total solar irradiation (TSI) is the primary source of energy that is provided to the Earth's climate system. A variation in the TSI irradiation will contribute to a natural climate variation on the Earth. The variability of

5 the irradiation from the Sun was approximately $0.3 \%$ over the last 300 years 
(Scafetta \& Willson, 2014). A better understanding of the TSI variability properties is critical for understanding the cause of the irradiation variability from the Sun. A TSI data series has information that reflects the cause of the TSI variability. If the TSI variability has deterministic oscillating periods, we can forecast expected TSI variation, whereas a random TSI variability can only explain the past. The intermittency of the solar variation is preferably explained as stochastic noise (Charbonneau, 2010). In this investigation, we introduce a simple hypothesis: if the TSI variability has a periodic oscillation, the variability oscillation must have an oscillation source that influences the solar energy oscillation. A possible oscillation source is the variable distance to the planets, which create oscillating tidal effects which we name gravity oscillations (GO).

\subsection{Solar variability}

The concept of a perfect and constant Sun, as postulated by Aristotle, was a strong belief for centuries and an official doctrine of Christian and Muslim countries (Usoskin et al., 2013). Although some transient changes of the Sun were observed with the naked eye, the introduction of the telescope in approximately 1600 demonstrated that the Sun had spots that varied in number and location. From 1610 systematic observations were reported. A pattern of sunspot variations was established when Heinrich Schwabe began regular observations of sunspots in 1826. He reported a possible period of approximately ten years (Schwabe, 1844). Wolf (1859) presented the opinion that the planets Venus, Earth, Jupiter and Saturn modulate the solar variability.

The solar activity cycle (Hathaway, 2015) consists of dark sunspots and bright regions (faculae) in addition to active regions that display sudden energy 30 releases (flares). The average cycle length is 11.1 years. During a cycle, the number of spots increases to a maximum number and then decreases. The average lifetime of a sunspot is slightly longer than the solar rotation period. They are bipolar, with the same magnetic polarity that leads with respect to the direction of the solar rotation. When the next cycle starts, spots appear 35 with opposite polarity at high latitudes in both hemispheres, and as the cycle 
progress, they appear closer to the Equator.

The 11.1-year sunspot period is referred to as the Schwabe cycle, and is proposed to be created by the tidal torque from the planets Venus, Earth and Jupiter (Wilson, 2013). The 22-year magnetic reversal period is referred to as

40 the Hale period. Scafetta (2012) showed that the 11-year Schwabe sunspot cycle consists of three periods of $9.98,10.90$ and 11.86 years, which are close to the Jupiter/Saturn spring period of 9.93 years, a tidal pattern of Venus, Earth and Jupiter of 11.07 years and the Jupiter orbital period of 11.86 years. A relation between the planets periods and sunspot periods indicates the possibility of a deterministic long-term relation between planet periods and periods in sunspot data series.

\subsubsection{Sunspot data series}

The sunspot number time series is a measure of the long-term evolution of the solar cycle and a proxy for the long-term influence of the Sun on the Earth's climate. The relative sunspot number $(R)$ as defined by Wolf $(1861)$ is based on the total number of individual sunspots $n$ and the number of sunspot groups $g$, according to the formula $R=k(10 g+n)$, where $k$ is a correction factor for the observer. It was introduced to correct for the use of different telescopes and observers. $R$ is referred to as the Zürich, Wolf or International Sunspot

${ }_{55}$ Number. Today $S N$ is used for the International Sunspot Number Clette et al. 2014).

Rudolf Wolf started systematic observations of sunspot numbers in 1849 . He also collected previous observations to construct daily sunspot numbers from 1750 and a yearly series from 1700. The cycle that started in 1755 became sunspot cycle 1 . The sunspot numbers had to be scaled upwards several times due to missing spots. By approximately doubling the number of recovered pbservations and cleverly interpolating between sparse observations (Hoyt et al., 1994), gaps were reduced and the series was extended to the first recording of sunspots by telescope in 1611. The history of the sunspot series and the last

${ }_{65}$ extensive corrections are described by Clette et al. (2014). The revised yearly 
series, which is available from the World Data Center SILSO from July 2015, was employed in our analysis.

Because the standard sunspot series is a composite time series based on single spots and groups, the accuracy significantly decreases going back in time. Due to poorer telescopes and locations, smaller spots were difficult to see and frequently lost. To correct for this situation, Hoyt and Schatten (1998a|b) constructed a new group sunspot number $R_{G}$ that was normalized to the Zürich sunspot number. Their series covered the period 1610-1995 and was based on a larger and more refined observational database. Although the group sunspot number corresponded to the relative sunspot number in the 20th century, the maximum group number was $40 \%$ lower in the 19th century and previous centuries (Clette et al., 2014). The group sunspot numbers were recently revised, and the difference between the series may now be considered as random noise. However, during the last two sunspot cycles (nos. 23 and 24), 30\% fewer sunspots per group were observed, which may be a sign of changes in the solar dynamo (Clette et al. 2014).

\subsubsection{Solar activity periods - grand maxima and minima}

In the 1890s, G. Spörer and E. W. Maunder (Maunder, 1890) reported that the solar activity was strongly reduced over a period of 70 years from 1645 to 851715 (Eddy, 1976, 1983). Based on naked-eye observations of sunspots, records of aurora activity, and a relation between ${ }^{14} \mathrm{C}$ variations and solar activity, a grand maximum (1100-1250) and the Spörer minimum (1460-1550) were also identified (Eddy, 1976).

The distribution of the solar activity can be interpreted as bi-modal, which implies distinct modes of activity. The main (regular) mode corresponds to moderate activity, which has a maxima of the 10-yr average spot number between 20 spots and 67 spots. In addition, we obtain grand maxima and grand minima that are above this range and below this range, respectively Usoskin et al., 2014). Studies that employ cosmogenic isotope data and sunspot data

95 indicate that we are currently leaving a grand activity maximum, which started 
in approximately 1940 and is now declining (Usoskin et al. 2003 Solanki et al. 2004 Abreu et al. 2008).

Because grand maxima and minima occur on centennial or millennial timescales, they can only be investigated using proxy data, i.e., solar activity reconstructed from ${ }^{10} \mathrm{Be}$ and ${ }^{14} \mathrm{C}$ time-calibrated data. The conclusion is that the activity level of the Modern Maximum (1940-2000) is a relatively rare event, with the previous similarly high levels of solar activity observed 4 and 8 millennia ago (Usoskin et al. 2003). Nineteen grand maxima have been identified by Usoskin et al. (2007) in an 11,000-yr series.

Grand minimum modes with reduced activity cannot be explained by only random fluctuations of the regular mode (Usoskin et al., 2014). They can be characterized as two flavors: short minima in the length range of 50-80 years (Maunder-type) and longer minima (Spörer-type). Twenty-seven grand minima are identified with a total duration of 1900 years, or approximately $17 \%$ of the time during the last 11,500 years (Usoskin et al., 2007). An adjustment-free reconstruction of the solar activity over the last three millennia confirms four grand minima since the year 1000: Maunder (1640-1720), Spörer (1390-1550), Wolf (1270-1340) and Oort (1010-1070) (Usoskin et al., 2007). The Dalton minimum (1790-1820) does not fit the definition of a grand minimum; it is more 115 likely a regular deep minimum that is observed once per century or an immediate state between the grand minimum and normal activity (Usoskin et al., 2013).

Temperature reconstructions for the last millennium for the northern hemisphere (Ljungquist, 2010) show a medieval maximum temperature at approximately the year 1000 and a cooling period starting at approximately 1350, immediately after the Wolf minimum and lasting nearly 500 years, with the coldest period in what is referred to as the Little Ice Age (LIA) at the time of the Maunder minimum. A cold period was also observed during the time of the Dalton minimum. The Maunder and the Dalton minima are associated with less solar activity and colder climate periods. In this investigation, minimum solar activity periods may serve as a reference for the identified minimum irradiations in the TSI oscillations. 


\subsection{Total Solar Irradiance}

The total solar irradiance (TSI) represents a direct index for the luminosity of the Sun measured at the average distance of the Earth. The solar luminosity blocking and faculae brightening against satellite TSI observations (Fröhlich \& Lean, 1998). To construct a TSI from a previous time period, two different approaches are employed: a reconstruction that is based on several different proxies for the solar irradiance (ACRIM-HS) or a statistical approach (PMOD).

sunspot structure, the decay rate of individual sunspots, the number of sunspots without umbra, the length and decay rate of the sunspot cycle, and the solar 
activity level.

Hoyt \& Schatten (1993) constructed an irradiance model that was based on

${ }_{\square}$ TSI reconstruction that was calibrated by the ACRIM TSI composite (Velasco Hererra et al., 2015). 


\subsection{Solar energy oscillation}

An oscillating TSI variability is produced by forcing from an oscillating energy source. This oscillation energy source may be the solar inertial motion, processes in the interior of the Sun, solar tides and/or solar orbit oscillation around the solar system barycenter (SSB). The energy source for the solar activity is the deceleration of the rotation of the Sun by magnetic field lines connected to interplanetary space. The solar wind carries mass away from the Sun; this magnetic braking causes a spin down of the solar rotation. Part of the decrease in rotational energy is the energy source for the solar dynamo, which converts kinetic energy to electromagnetic energy.

The classical interpretation of the solar dynamo is that it is placed in the transition zone between convection and radiation near the solar surface: the tachocline, approximately 200,000 km below the surface. Strong electric currents originate by the interaction between the convection and the differential solar rotation. This causes the formation of strong magnetic fields, which rise to the surface and display various aspects of solar activity, such as spots, facular fields, flares, coronal mass emissions, coronal holes, polar bright points, and polar faculae, after having detached, as described by de Jager \& Duhau (2011). They explain the 22-year Hale cycle as attributed to magneto-hydrodynamic oscillations of the tachocline. This period is not constant. It persisted for approximately 23 years prior to the Maunder Minimum, during which it increased to 26 years. During the maximum of the last century, this period was as brief as 21 years. Gleissberg (1958, 1965) discovered a cycle of approximately 80 years in the amplitude of the sunspot numbers. It is interpreted as the average of two frequency bands: one band from 50-80 years and one band from 90-140 years Ogurtsov et al., 2002). An examination of the longest detailed cosmogenic isotope record (INTCAL98) of ${ }^{14} \mathrm{C}$ abundance, with a length of 12,000 years, reveals an average Gleissberg cycle period of 87.8 years. It is resolved in two combination periods of $91.5 \pm 0.1$ and $84.6 \pm 0.1 \mathrm{yr}$ (Peristykh \& Damon, 2003).

Proxies that describe the magnetic fields in the equatorial and polar regions can describe the variability of the tachocline. A proxy for the equatorial 
(or toroidal) magnetic field is $R_{\max }$ (the maximum number of sunspots in two successive Schwabe cycles), and a proxy for the maximum poloidal magnetic field strength is $a a_{\text {min }}$ (the minimum value of the measured terrestrial magnetic field difference). In a phase diagram based on the $R_{\max }$ and $a a_{\min }$ values, two Gleissberg cycles (1630-1724) and (1787-1880) were identified (Duhau \& de

225 Jager, 2008). The years 1630 and 1787 represent transition points, where phase transitions to the grand episodes (Maunder and Dalton minima) occurred. The lengths of the two Gleissberg cycles were 157 years and 93 years. The next Gleissberg cycle lasted 129 years until 2009 with an expected phase transition to a high state in 1924. Duhau \& de Jager (2008) predicted that the transition in 2009 indicates a transition to a Maunder-type minimum that will start with cycle 25 in approximately 2020.

In addition to the variable Gleissberg period, a de Vries period from 170-260 years is observed in the ${ }^{14} \mathrm{C}$ and ${ }^{38} \mathrm{Cl}$ records. This period is fairly sharp with little or no variability (Ogurtsov et al. 2002). Almost no existing models for the solar activity predicted the current weak cycle 24. A principal component analysis of full disc magnetograms during solar cycles 21-23 revealed two magnetic waves that travel from opposite hemispheres with similar frequencies and increasing phase shifts (Shepherd et al. 2014, Zharkova et al., 2015). To understand this phase shift they introduce a non-linear dynamo model in a two-layer medium with opposite meridional circulation. One dynamo is located in the surface layer and the other dipole deeply in the solar convection zone. The solar poloidal field is generated by these two dynamos in different cells with opposite meridional circulation. The observed poloidal-toroidal fields have similar periods of oscillation with opposite polarities that are in an anti-phase every lation flow is detected with helioseismology by HMI/SDO observations (Zhao et al. 2013). Extrapolations backward of these two components revealed two 350 -year grand cycles that were superimposed on a 22-year cycle. The beat between the two waves shows a resemblance to the sunspot activity, including the Maunder and Dalton minima, and forecasts a deep minimum in this century. 
The low frequency wave has a variable period length from 320 year (in 18-20 centuries) to 400 year predicted for the next millennium.

Analysis of systematic variations of small scale magnetic structures and EUV bright spots from the Solar and Heliospheric Observatory (SOHO) and Solar Dyin the convection zone is on the order of $10^{5}$ years (Foukal et al. 2009), which is too long to explain the rapid decay of the magnetic field during one solar cycle. A simple solution is to place the dynamo in small bubbles in the solar core, which change polarity every cycle due to interaction with the interplanetary magnetic 
on the solar surface as precursors for large flares. The largest flares have a high probability of appearing near the closest position of one or more of the tide-producing planets Mars, Venus, Earth and Jupiter (Hung, 2007, Mörner et

285 al. 2015). The energy of the hot bubbles is boosted by thermonuclear runaway processes in the bubbles, which appear at the solar surface as hot areas with a frozen magnetic field. In this process, planetary effects serve an important role (Granpierre, 1990, 1996, Wolf \& O'Donovan, 2007, Scafetta, 2012).

\subsection{External forcing generated by the planets}

Although the various dynamo models can explain the variations to some extent, few or no constraints on the periods exist. The majority of the explanations operate with a range of possible periods. The models do not explicitly determine whether the observed periods are random and stochastic or if some period-forcing from external or internal sources occurs. In the following section, we investigate the external forcing that is generated by the planets in the solar system.

\subsubsection{Solar inertial motion}

Charvátová \& Heida (2014) have classified the solar inertial motion (SIM) in an ordered (trefoil) pattern with a length of approximately 50 years followed by disordered intervals. Exceptionally long (approximately 370 years) trefoil patterns appear with a 2402-year Hallstadt period. They determined that the deepest and longest solar activity minima (of Spörer and Maunder types) appeared in the second half of the 2402-year cycle, in accordance with the most disordered type of SIM. The Dalton minimum appeared during a mildly disordered SIM (1787-1843), which repeats from 1985-2040. The solar orbit in the period 1940-2040, which is shown in Figure 1, demonstrates this phase.

\subsubsection{Interior of the Sun as a rotating star}

Wolf \& Patrone (2010) have investigated how the interior of a rotating star can be perturbed when the star is accelerated by orbiting objects, as in the solar system. They present a simple model in which fluid elements of equal 
mass exchange positions. This exchange releases potential energy (PE) that is only available in the hemisphere that faces the barycenter of the planetary system, with a minor exception. This effect can raise the PE for a few wellpositioned elements in the stellar interior by a factor of 7 , which indicates that a star with planets will burn nuclear fuel more effectively and have a shorter lifetime than identical stars without planets. However, in the case of the Sun, occasional mass exchanges occur near the solar center, which activate a mixed shell situated at $0.16 r_{s}$ where $r_{s}$ is the solar radius. For this reason, the close passages of the barycenter are important because they can cause negative pulses in the PE. The energy liberated is a result of the roto-translational dynamics of the cell around the solar system barycenter. An analysis of the variation of the PE storage reveals that the maximum variations correspond to the documented grand minima of the last 1000 years because the PE minima are connected to periods in which the Sun moves close to the barycenter. Large reductions in the PE values occur when the giant planets are quasi-aligned, which occurred in approximately 1632, 1811 and 1990, separated by 179 years (Jose cycle). Because the planetary positions never exactly repeat, the PE variations show a complex pattern that creates different minima (Cionco \& Soon, 2015).

\subsubsection{Solar inertial oscillations}

The complex planetary synchronization structure of the solar system has been known since the time of Pythagoras of Samos (ca. 570-495 B.C.). Jose (1965) showed that the solar center moves in loops around the solar system barycenter (SSB). The average orbital period of 19.86 years corresponds to the heliocentric synodic period of Jupiter and Saturn. The modulation of the orbit by the outer planets Uranus and Neptune produces asymmetries in the prbital shape and period variations between 15.3 and 23.4 years (Fairbridge \& Shirley, 1987). The solar motion differs from the Keplerian motion of planets and satellites in important ways. For instance, the velocity is some time highest when the distance from the Sun to the SSB is largest, and the solar angular 
years (Blizzard, 1981). An analysis of solar orbits from A.D. 816 - 2054 covered seven complete Jose cycles of 179 years and indicated that prolonged minima can be identified by two parameters: the first parameter is the difference in axial symmetry of the orbit, and the second parameter is the change in angular momentum (torque) about SSB. Based on these criteria, a new minimum should begin between 1990 and 2013 and end in 2091 (Fairbridge \& Shirley, 1987).

The distance of the Sun from the barycenter, the velocity, and the angular momentum show the same periodic behavior. The motion of the solar center around the SSB is typically prograde; however, in 1811 and 1990, the Sun occasionally passes near the SSB in a retrograde motion. Because the 1811 event occurred at the time of the Dalton minimum, a new minimum may occur in approximately 1990 (Cionco \& Soon, 2015).

Scafetta (2014) reviews the investigation of the patterns that are described by the Sun and planets. He concludes that modern research shows that the planetary orbits can be approximated by a simple system of resonant frequencies and that the solar system oscillates with a specific set of gravitational frequencies, many of which range between three and 100 years, that can be constructed as harmonics of a base period of $\sim 178.78$ years.

\subsubsection{Solar tidal oscillation}

The tidal elongation at the solar surface is on the order of 1-2 $\mathrm{mm}$ from the planets Venus and Jupiter with less tides from the other planets. Scafetta (2012) proposed that tidal forces, torques and jerk shocks act on and inside the Sun and that the continuous tidal massaging of the Sun should involve heating the core and periodically increasing the nuclear fusion rate. This action would amplify weak signals from the planets with a factor $\sim 4 \times 10^{6}$. Even if the amplitude is small in the direction of a planet, it creates a wave that propagates with the velocity of the planet. If the planet has an elliptical orbit, the variation in distance creates a disturbance that will affect the nuclear energy production in the center of the Sun. Since more planets participate, this effect or GO, will be a combination of phases and periods, which can be highly nonlinear. 
Our hypothesis is that the solar position oscillation (SPO) represents an indicator of the tidal and inertial interaction between the giant planets Jupiter, Saturn, Neptune, Uranus and the Sun. The SPO can be calculated from planetary Ephemeris as the movement of the Sun around the Solar System Barycenter

375 (SSB). In section 2, we describe the methods and data sets used to demonstrate a connection between SPO and TSI and SN variations. In section 3, we present the results; in section 4 , we discuss the results and relate them to other investigations. We conclude the paper in section 5 . Because the solar system and its planets has a long lifetime, we can expect forces in the same direction over long periods of time that may have a strong effect on long periods.

\section{Materials and methods}

\subsection{Data}

The motive of the study is to identify possible stationary periods in TSI variability. In this study possible stationary periods are represented by first tionary periods have a period $T$ in the data series. Subharmonic periods have periods $n * T$ for $n=2,3,4 \ldots$ Coincidence periods have a coincidence between two ore more periods and may be represented by $n * T_{1}=m * T_{2}$. Coincidence periods are stationary periods and introduce a new set of subharmonic periods. TSI data series, a sunspot data series and a SPO data series.

The SPO data series represents an indicator of the oscillating tidal and inertial interaction between the Sun and the large planets. The large planets have the following periods (in years): Jupiter $P(J, 11.862)$, Saturn $P(S, 29.447)$, 1000 to 2100 , where $S P O x$ represents the $x$-direction of the $x y z$-vector. The source of the SPO data series is the JPL Horizon web interface (http://ssd.jpl.nasa.gov/horizons.cgi\#top is based on the Revised July 31, 2013 ephemeris with the ICRF/J2000 reference frame, downloaded 30.09.14 and at subsequent dates. 
The total solar irradiance (TSI-HS) data series (e.g. Scafetta \& Willson, 2014 Fig. 16) covers the period from 1700 to 2013. The source of the data series is Scafetta (personal communication. Dec. 2013). The total solar irradiation (TSI-LS) is based on the LS-SVM ACRIM data series (Velasco Hererra et al. 2015) and covers the period from 1000 to 2100. The source of the TSI-LS data series is Velasco Herrera (21.09.14. Personal communication). The sunspot data series is the group sunspot numbers from 1610 to 2015. The source of the sunspot data series is SILSO (The World Data Center for the production, preservation and dissemination of the international sunspot number).

\subsection{Methods}

Possible stationary periods in the data series are identified in two steps. First a wavelet transform of the data series separates all periods in into a wavelet spectrum. The autocorrelation for wavelet spectrum then identifies dominant first stationary periods, subharmonic periods and the coincidence periods. Prior to the wavelet analysis, all data series are scaled by

$$
x(t)=(y(t)-E[y(t)]) / \operatorname{var}(y(t))
$$

415

where $y(t)$ is the data series, $E[y(t)]$ is the mean value, $\operatorname{var}(y(t))$ is the variance and $x(t)$ is the scaled data series. The data series are scaled to compare the amplitudes from the oscillation periods and reduce side effects in the wavelet analysis.

A wavelet transform of a data series $x(t)$ has the ability to separate periods in the data series into a wavelet spectrum. The wavelet spectrum is computed by the transformation

$$
W_{a, b}(t)=\frac{1}{\sqrt{a}} \int_{R} x(t) \psi\left(\frac{t-b}{a}\right) d t
$$

where $x(t)$ is the analyzed time series, $\psi()$ is a coif3 wavelet impulse function (Daubechies, 1992, Matlab, 2015); which is chosen for its symmetrical performance and its ability to identify symmetrical periods in data series; $W_{a, b}(t)$ 
represents the computed wavelet spectrum, the parameter $a$ represents a timescaling parameter, and the parameter $b$ represents a translation in time in the wavelet transformation. When $b=0$ and $s=1 / a$, the wavelet spectrum $W(s, t)$ represents a set of moving correlations between $x(t)$ and the impulse function $\psi()$ over the entire time series $x(t)$. The relationship between the wavelet $s$ and a sinus period $T$ is approximately $T \sim 1.2 \mathrm{~s}$ when using the coif3 wavelet function. In this investigation, the wavelet spectrum $W(s, t)$ has the spectrum range $s=0,1,2 \ldots 0.6 N$, where $N$ is the number of samples in the data series.

An autocorrelation transformation of the wavelet spectrum $W(s, t)$ identifies first periods, subharmonic periods and coincidence periods as maximum values in the computed set of autocorrelation functions. The set of autocorrelation functions are estimated by the transformation

$$
R(s, m)=E[W(s, t) W(s, t+m)]
$$

where $R(s, m)$ represents the correlations between samples, at a distance $m$ years, for a wavelet $s$ in the wavelet spectrum $W(s, t)$.

\section{Results}

\subsection{Sun Position Oscillation}

The Sun moves in a closed orbit around the barycenter of the solar system. Figure 1 shows the SPO in the ecliptical plane from 1940 to 2040. The solar system oscillation (SSO) is caused by the mutual gravity dynamics between the planet system oscillation (PSO) and the solar position oscillation (SPO). The solar position oscillation has oscillations in the $(x, y, z)$ directions; they are represented by the data series $S P O x, S P O y$ and $S P O z$. The movement looks rather chaotic, as shown in Figure 1, because it mirrors the movements of the planets in their orbits. A first step in this investigation is to identify stationary periods and phase relations in the solar position between A.D. 1000 and 2100 .

A wavelet spectrum represents a set of moving correlations between a data series and a scalable wavelet pulse. When the data series in the y-direction 
- SPOy - is transformed to the wavelet spectrum $W \operatorname{spoy}(s, t)$, the spectrum represents a collection of dominant periods in the SPOy data series. A visual inspection of the wavelet spectrum $W \operatorname{spoy}(s, t)$ shows a long-term dominant period of approximately Pspoy(164) years. This period has a coincidence to the Neptune period $\mathrm{P}(\mathrm{N}, 164.79)$. The data series $S P O x$ and $S P O y$ have the same periods; however, $S P O y$ has a 90 -degree phase delay with respect to $S P O x$.

The wavelet spectrum $W \operatorname{spoy}(s, t)$ is transformed to a set of autocorrelation functions Rspoy $(s, m)$, as shown on Figure 2, where each colored line represents a single autocorrelation function. The set of autocorrelations $R s p o y(s, t)$ shows the identified stationary periods in the wavelet spectrum $W \operatorname{spoy}(s, t)$. The first maximum represents the correlation to a first stationary period. Subharmonic periods have a maximum correlation at a distance (first period) $* n$ where $n=1,2,3 \ldots$ Rspoy $(s, m)$ identifies the following stationary periods: $P($ spoy, 12$)$ for $R \operatorname{spoy}(12)=0.98, P($ spoy, 29$)$ for $R \operatorname{spoy}(29)=0.95, P($ spoy, 84$)$ for Rspoy $(84)=0.9$ and $P($ spoy, 164$)$ for Rspoy $(164)=0.9$. The same periods are associated with the PSO periods $P(J, 11.862), P(S, 29.447), P(U, 84.02)$ and $P(N, 164.79)$, which indicates that the planets Jupiter, Saturn, Uranus and Neptune in the planetary system are controlling the SPO.

A coincidence between subharmonic periods will amplify the coincidence period and introduce a new set of stationary periods. The autocorrelation spectrum Rspoy $(s, m)$ of Figure 2 shows a set of subharmonic periods - $P(s p o y, n *$ 12), $P($ spoy, $n * 29)$ and $P($ spoy, $n * 84)$ - where $n=1,2,3 \ldots$. The identified coincidence periods have mean values of

${ }_{475}(P($ spoy, $5 * 12)+P($ spoy, $2 * 29)) / 2=P($ spox, 59$)$ for $R W($ spoy, 59$)=0.95$, $P($ spoy, $7 * 12)+P($ spoy, $3 * 29)+P($ spoy, 84$)) / 3=P($ spoy, 85$)$ for $R W($ spoy, 85$)=$ 0.9 $(P($ spoy, $10 * 12)+P($ spoy, $4 * 29)) / 2=P($ spoy, 118$)$ for $R \operatorname{spoy}(118)=0.9$ and $(P($ spoy, $2 * 84)+P($ spoy, 164$)) / 2=P($ spoy, 166$)$ for $\operatorname{Rspoy}(166)=0.9$.

480 Figure 2 reveals that the majority of the SPO periods are mutually related by resonance. The new modulated periods are $P($ spoy, 59) and $P($ spoy, 118).

The stationary long wavelet periods $W$ spoy $(84, t)$ and $W \operatorname{spoy}(164, t)$ have 
maxima in approximately 1820 , and $W \operatorname{spoy}(29, t)$ has a maximum in approximately 1812. The identified stationary periods may be transformed to the model:

$$
\begin{array}{r}
P(\text { spoyc }, 29, t)=R W(\text { spoy }, 29) \cos (2 \pi(t-1812)) / 29.447) \\
P(\text { spoyc }, 84, t)=R W(\text { spoy }, 85) \cos (2 \pi(t-1820)) / 84.02) \\
P(\text { spoyc }, 164, t)=R W(\text { spoy }, 164) \cos (2 \pi(t-1820)) / 164.97)
\end{array}
$$

By this model, the year 1820 may serve as a phase reference for the SPOy periods, TSI variability and solar variability (SN). The data series $S P O x$ has the same stationary periods but a different phase. $P(\operatorname{spoxc}, 84, t)$ has a maximum at approximately 1797 , which represents a phase shift of approximately $\pi / 2$. $P($ spoxc $, 164, t)$ has a maximum at approximately 1779 . The maxima in $S P O y$ and $S P O x$ corresponds to minima in $S P O \ddot{y}$ and $S P O \ddot{x}$. The deterministic model (Eq. 4-6) has the sum $P($ spoyc, $29, t)+P($ spoyc, $84, t)+P($ spoyc $, 164, t)$ and a maximum in approximately 1812 .

\subsection{TSI-HS variability}

The total solar irradiation (TSI) represents the measured irradiation $\mathrm{Wm}^{-2}$ at the average distance from the Sun to the Earth. Figure 3 shows an annual mean total solar irradiance (TSI-HS) data series (Scafetta \& Willson, 2014) that covers the period from A.D. 1700 to 2013. A simple visual inspection of this data series shows some variability properties. The TSI-HS data series irradiation has fluctuations of approximately $3-4 \mathrm{Wm}^{-2}$. The TSI fluctuations have minima in approximately 1700 (or before), 1800, 1890, and 1960, with gaps of approximately 100, 90, and 70 years, or a mean minimum period of approximately 86 years. The TSI-HS data series has maxima in 1770, 1830, and 1950, with gaps of approximately 60 and 120 years. The mean maximum fluctuation period in the TSI-HS data series is approximately 75 years or 11 years less than the mean minimum period. Transformation of the TSI data series into a wavelet spectrum may identify stationary periods. 
The transformed wavelet spectrum $W h s(s, t)$ represents a set of separated wavelet periods from the TSI-HS data series. Figure 4 shows the computed wavelet spectrum of the TSI-HS data series from 1700 to 2013. In this presentation, the wavelet scaling range is $s=1 \ldots 0.6 \mathrm{~N}$, and the data series contains $N=313$ data points. A visual inspection of the TSI wavelet spectrum shows the dominant periods in the TSI data series in the time window between 1700 and 2013. The long wavelet period has a maximum in 1760, 1840, 1930, and 2000 , with a mean gap of approximately 80 years.

The autocorrelation spectrum $R h s(s, m)$ of the wavelet spectrum $W h s(s, t)$ identifies stationary periods in the wavelet spectrum. The maximum values in the autocorrelation spectrum $R h s(s, m)$ represent a correlation to stationary periods in the TSI-HS wavelet spectrum. Figure 5 shows the autocorrelation ${ }_{520}$ spectrum $R h s(s, m)$ of the wavelet spectrum $W h s(s, t)$ of the TSI-HS data series.

A study of the autocorrelation spectrum $R h s(s, m)$ shows a set of stationary periods in the $W h s(s, t)$ wavelet spectrum. The identified first cause stationary periods comprise the period set $P(h s, 11)$ for $R h s(11)=0.55, P(h s, 49)$ for ${ }_{525} \operatorname{Rhs}(49)=0.55, P(h s, 86)$ for $R h s(86)=0.65$ and $P(h s, 164)$ for $R W h s(164)=$ 0.7. The identified stationary periods are associated with the PSO periods $P(J, 11.862), P(S, 29.447), P(U, 84.02)$ and $P(N, 164.79)$. The identified stationary period $P(h s, 49)$ may be explained by possible modulation between the Saturn oscillation and the Neptune oscillation: $2 /(1 / P(S, 29.447)+1 / P(N, 164.79))=$ $P(S, N, 49.96)$. This finding indicates that the TSI-HS variability is related to the solar position oscillation, which is controlled by the planet oscillation from the large planets Jupiter, Saturn, Uranus and Neptune. Additional analysis indicates that the dominant wavelet periods $W h s(11, t)$ and $W h s(49, t)$ are mean estimates. Whs $(11, t)$ has phase disturbance and $W h s(49, t)$ has a phasereversal, as shown in Figure 6. They do not have stable phases and represent mean periods.

Figure 6 shows the identified dominant stationary wavelet periods $W h s(49, t)$, $W h s(86, t)$ and $W h s(165, t)$ from the autocorrelation functions in $R h s(s, m)$. It 
shows that the wavelet period $P(h s, 49)$ has a time-variant phase and is not a stable period. The TSI-HS wavelet periods $W h s(49, t)$ and $W h s(86, t)$ have a negative value coincidence in the period from 1786 to 1820 . $W \operatorname{spoy}(84, t)$ has an estimated maximum velocity and $W \operatorname{spox}(84, t)$ has maximum state at approximately 1797 . The dominant wavelet period $W h s(84, t)$ has a minimum state at approximately 1803, or a phase delay of approximately $0.15 \pi$ ( rad/yr)

between the $W \operatorname{spox}(84, t)$ maximum and the minimum $W h s(84, t)$. Uranus was in perihelion in 1798. This indicates a relation between a minimum Uranus distance to the Sun and a minimum in TSI-HS.

The correlation between the TSI-HS data series and the identified dominant wavelet periods $W h s(49, t)+W h s(86, t)+W h s(164, t)$ is estimated to be ${ }_{550} R=0.93, Q=46.6$ (Pearson correlation coefficient) in $N=312$ samples. The correlation $R=0.93$ reveals a close relation between the TSI-HS variability and the solar position oscillation, which is controlled by Jupiter, Saturn, Uranus and Neptune.

\subsubsection{Deterministic model}

The identified stationary periods $W h s(86, t)$ and $W h s(164, t)$ may be represented by a deterministic model from the sum of the stationary cosine functions:

$$
\begin{array}{r}
P(h s c, 84, t)=-R h s(86) \cos (2 \pi(t-1803) / 84.02) \\
P(h s c, 164, t)=-R h s(164) \cos (2 \pi(t-1860) / 164.97) \\
P(h s c, t)=P(h s c, 84, t)+P(h s c, 164, t)
\end{array}
$$

where $R(h s, 86)$ and $R(h s, 164)$ represent estimated correlations in the autocorrelation. The phase relation between the maximum value of $P \operatorname{spox}(84, t)$ in 1797 and the minimum value $P(h s c, 84, t)$ in 1803 is approximately $0.15 \pi$ 560 (rad/year). The year 1797 is also the year of Uranus in perihelion. We also notice that the minimum value of $P(h s, 164, t)$ (Eq. 8) is close to the time of Neptune in perihelion. This indicates a delayed response from the SPO periods on the TSI-HS variability. The correlation between the 84-year wavelet periods $P(h s c, 84, t)$ and $W(h s c, 84, t)$ is estimated to be $R h s, h s c(84)=0.83$. The 

periods in the TSI-HS data series, the SPO data series periods and the PSO periods $P(J, 11.862), P(S, 29.447)$ and $P(U, 84.02)$. The difference is the smaller correlation value in the autocorrelation $\operatorname{Rls}(s, m)$. Smaller correlation values 


\subsubsection{Deterministic model}

The identified dominant periods $W l s(125, t), W l s(210, t)$, and $W l s(373, t)$ may be represented by the deterministic stationary model from the sum of the cosine functions

$$
P(l s c, 126, t)=R l s(125) \cos (2 \pi(t-1857) /(3 * 84.02 / 2)
$$




$$
\begin{aligned}
& P(l s c, 210, t)=R l s(210) \cos (2 \pi(t-1769) /(5 * 84.02 / 2) \\
& P(l s c, 378, t)=R l s(373) \cos (2 \pi(t-1580) /(9 * 84.02 / 2) \\
& P(l s c, t)=P(l s c, 126, t)+P(l s c, 210, t)+P(l s c, 378, t)
\end{aligned}
$$

where $R l s(125), R l s(210)$ and $R W l s(373)$ represent the maximum period corre-

$P(l s c, t) \leq-1.0$. The identified minima from this model are $P(l s c, t) \leq-1.0$ for the time period (1014-1056); $P(l s c, t) \leq-0.5)$ for $(1276-1301) ; P(l s c, t) \leq-1.0$ for (1404-1435), which has a minimum -1.215 in the year 1419; $P(l s c, t) \leq-0.5$ for (1662-1695) which has a minimum -0.91 in the year 1672 ; and $P(l s c, t) \leq$ $650-0.5$ for (1775-1819), which has a minimum -0.81 in the year 1796 . The com- 
puted subsequent minimum time period is $P(l s c, t) \leq-0.5$ for (2035-2079), which has a minimum -0.79 in the year 2057. In this model, a Dalton-type minimum has a minimum state at approximately -0.7 . The Maunder minimum is between -0.7 and -1.0 , as shown in Table 2 . The computed minimum -0.79 in the year 2057 indicates an expected Dalton-Maunder-type minimum. The deterministic model has a state $P(l s c, t) \geq+0.5$ index for the periods (1093-1134), (1198-1241) and (1351-1357); $P(l s c, t) \geq+1.0$ index for the period (1582-1610); $P(l s c, t) \geq+0.5$ for (1945-2013); and $P(l s c, t) \geq+1.0$ for (1959-2001), which has a maximum 1.4 in 1981.

\subsection{Sunspot variability}

The sunspot data series $S N(t)$ is an indicator of the solar variability. Figure 10 shows the group sunspot number data series that covers a period of approximately 400 years from A.D. 1610 to 2015. From this 400-year data series, we can estimate periods of approximately up to 200 years. Periods with few sunspots are associated with low solar activity and cold climate periods. Periods with many sunspots are associated with high solar activity and warm climate periods. If a relation exists between solar periods and climate periods, we may expect a relation between the periods in the TSI variability and solar variability.

Figure 11 shows the computed wavelet spectrum $W s n(s, t)$ of the $S N(t)$ data series from 1610 to 2015 , with the wavelet scaling parameter $s=1 \ldots 6 N$. A visual inspection of the wavelet spectrum shows a maximum at the approximate years $(1750,1860,1970)$, which represents periods of approximately 110 years. The time from 1750 to 1970 represents a period of 220 years. Temporary periods of approximately 50 years from approximately 1725 and 1930 may be confirmed by computing the autocorrelation wavelet spectrum $R s n(s, t)$.

The computed set of autocorrelations $R s n(s, m)$ of the wavelet spectrum $W s n(s, t)$ is shown in Figure 12. The wavelet spectrum $W(s n, t)$ has the stationary periods $P(s n, 11)$ for $R s n(11)=0.73, P(s n, 22)$ for $R s n(22)=0.35$ and $P(s n, 86)$ for $R s n(86)=0.35$. The identified period $P(s n, 11)$ represents the Schwabe cycle and corresponds to the TSI $P(t s i, 11)$, the SPO period 
$P($ spox, 11$)$ and the Jupiter period $P(J, 11.862)$.

The period $P(s n, 11)$ introduces the subharmonic period $P(s n, 5 * 11)=$ $P(s n, 55)$ for $R s n(55)=0.43$, which introduces the subharmonic periods $P(s n, 110)$ for $\operatorname{Rsn}(110)=0.40$ and $P(s n, 210)$ for $R s n(210)=0.36$. The period $P(s n, 55)$ is a temporary stationary period from 1610 only when $P(s n, 110)$ has a positive state. An inspection of $P(s n, 55)$ shows that the period is stationary when $P(s n, 210)$ has a positive state from $1726-1831$ and from 1935 . The period $P(s n, 55)$ shifted to $P(s n, 2 * 55)$ when $P(s n, 210)$ has a negative state from 18311935. A possible explanation is a $5 / 2$ relation between the periods $P(U, 84.04)$ and $P(s n, 210)$ (Eq. 16).

Figure 12 shows that the period $P(s n, 55)$ has combination resonance periods with a $3 / 2$ relation $P(s n, 3 * 55 / 2=84)$ to the Uranus period $P(U, 84.02)$. The $3 / 2$ correlation to the $P(l s, 84)$ period and the Uranus period $P(U, 84.02)$ explains the synchronization between the SN variability and the TSI-LS variability. The dominant period $P(s n, 110)$ is a coincidence period in the subharmonic period $P(s n, 2 * 55=110)$, which has a combination resonance to the Neptune period by $P(s n, 2 * 164,79 / 3=109,86)$. The long stationary identified period $P(s n, 210)$ is related to a $5 / 2$ combination resonance to Uranus by $P(U, 5 * 84.02 / 2=210.05)$. The period $P(s n, 210)$ corresponds to the TSILS period $P(l s, 210)$. The identified periods have a subharmonic resonance in the Jupiter period $P(J, 11.862)$. The correlation between the data series $\mathrm{SN}(\mathrm{t})$ and the dominant wavelet periods $W(s n, 55, t)+W(s n, 110, t)+W(s n, 210, t)$ is estimated to be $R=0.51$ for $\mathrm{N}=404$ and $\mathrm{Q}=11.8$.

\subsubsection{Deterministic model}

The identified temporary stationary periods $W s n(55, t), W \operatorname{sn}(110, t)$ and $W l s(210, t)$ may be represented by a deterministic model

$$
\begin{array}{r}
P(\text { snc }, 56, t)=R \operatorname{sn}(55) \cos (2 \pi(t-1782) /(2 * 84.02 / 3) \\
P(\text { snc }, 112, t)=R \operatorname{sn}(110) \cos (2 \pi(t-1751) /(4 * 84.02 / 3) \\
P(\text { snc }, 210, t)=R \operatorname{sn}(210) \cos (2 \pi(t-1770) /(5 * 84.02 / 2)
\end{array}
$$




$$
P(\text { snc }, t)=P(\text { snc }, 56, t)+P(\text { snc }, 112, t)+P(\text { snc }, 210, t)
$$

where $R s n(55), R s n(110)$ and $R s n(210)$ represent the maximum correlation in the autocorrelation $R s n(s, m)$. This model is, however, a simplified linear model. Figure 11 shows that the $R s n(55)$ amplitude is controlled by the $R s n(110)$ amplitude, which indicates that the period $P(s n, 55)$ is temporarily stable. The correlation between the 55 -year wavelet periods $W(s n, 55, t)$ and the stationary period $P(s n c, 55, t)$ is estimated to be $R s n, \operatorname{snc}(55)=0.66$ for $\mathrm{N}=354$ samples and $\mathrm{Q}=16.6$. The correlations are $R s n$, snc(110) $=0.9$ for $\mathrm{N}=304$ and $\mathrm{Q}=36$ and $\operatorname{Rsn}, \operatorname{snc}(210)=0.9$ for $\mathrm{N}=304$ and $\mathrm{Q}=36$. The correlation between the sum $W(s n, 55, t)+W(s n, 110, t)+W(s n, 210, t)$ and the deterministic model from (Eq. 17) is estimated to be $R=0.84$ for $N=304$ and $Q=29.8$. Minimum states that correspond to negative values of the stationary model correspond to the observed minima, as shown in Table 2. The model indicates a future minimum in the period 2018 - 2055 with an extreme value in 2035.

This analysis indicates that the sunspot variations is controlled by the Uranus period $P(U, 84.02)$, which introduces a $2 / 3$ resonance to the period $P(s n, 55, t)$ and a $5 / 2$ resonance to the $P(s n, 210)$ period. The TSI-LS data series and the sunspots data series have stationary coincidence periods with $P(l s, 11)$ and $P(s n, 11), P(l s, 125)$ and $P(s n, 110)$ and with $P(l s, 210)$ and $P(s n, 210)$. The difference between the stationary periods $P(l s, 125)$ and $P(s n, 110)$ indicates a limited direct relation between the data series.

\subsection{Stationary dominant periods and minima}

The relations between the identified dominant periods in the SN, TSI-HS and TSI-LS series are shown in Table 1 , where $R$ is the autocorrelation of the wavelet spectrum.

In Table 2 we compare values of the stationary models $P(h s c, t), P(l s c, t)$ and $P(s n c, t)$ at minima corresponding to the solar activity minima determined by Usoskin et al. (2007). The grand minimum periods are calculated from the stationary models in Equations 9, 13 and 17, and compared with Spox and Spoy 

approximately 2025 to 2050; the model $P(h s c, t)$ computes a new Dalton TSI minimum period (2035-2065), and the model $P(l s c, t)$ computes a new Dalton TSI minimum period (2045-2070).

The SN model in Eq. 17 is a simplified linear model. It has a minimum $P($ snc,$t) \leq-0.5$ in $1907-1931$, which is not shown in the table. The HS-model from Eq. 9 has grand minima in 1200-1230 and 1876-1887, which are not shown in the table. For this model the Dalton minimum is less deep. The LS-model from Eq. 13 has the maximum index $P(l s c, t) \geq 0.5$ for the periods (10931134), (1198-1241), (1351-1357), and (1945-2013) and the grand maximum index $P(l s c, t) \geq+1.0$ for the periods (1582-1610) and (1959-2001).

\section{Discussion}

The study of the TSI variability is based on the TSI-HS data series from 1700-2013, the TSI-LS data series 1000-2100, sunspots data series 1610-2015 and a Solar Barycenter orbit data series from 1000-2100. The results are, however, limited by how well they represent the solar physics and how well the methods are able to identify the periods in the data series. The investigation is based on a new method. The data series are transformed to a wavelet spectrum to separate periods, and the wavelets are transformed into a set of autocorrelations to identify the first periods, subharmonic periods and coincidence periods. The identified stationary periods in the TSI and SN series are supported by the close relations with the well-known solar position periods and documented solar minimum periods. The solar orbit data will then provide a stable and computable reference. We have used the Dalton minimum (1790-1820) as a reference period, since our two TSI-series and the SN-series cover this minimum. We notice that maxima in SPOx and SPOy corresponds to minima in SPO $\ddot{x}$ and $S P O \ddot{y}$ which means maximum negative acceleration. We use $P($ spoxc $, 84, \max )=1797$ as a time of reference. This is close to Uranus perihelion (1798) and Neptune aphelion (1804), which indicates a possible relation between the distance to these 
planets and the Dalton minimum.

\subsection{TSI-HS variability}

The dominant periods in the TSI-HS variability are related to the large planets, as shown in Table 1. The correlation between the TSI-HS data series and the identified dominant wavelet periods $W h s(49, t)+W h s(86, t)+W h s(164, t)$ is estimated to be $R=0.93$. The dominant periods $P(h s, 11)$ and $P(h s, 49)$ have a time-variant phase and represent mean estimates. A possible source of the $P(h s, 49)$ period is the interference between the Saturn period $P(h s, 29)$ and the Neptune period $P(h s, 164)$. The periods $P(h s, 84)$ and $P(h s, 164)$ have a stable period and phase in the time period from 1700 to 2013. The TSI-HS data series from 1700 is too short for a reasonable estimate of $P(h s, 164)$. A

possible alternative is a coincidence resonance between the subharmonic period $P(h s, 2 * 84=168)$ and the 164 year Neptune period.

The model $P(h s c, t)$ computes the deterministic oscillations in the TSI-HS variability. Table 2 shows that $P(h s c, t)$ computes a minimum in the period from 1796-1830. In the same time period, $P($ spox, 84$)$ and $P($ spoy, 84$)$ have maxima in 1797 and 1820. A close relation between the minimum of the period $P(h s, 84)$ and the maximum states of $P($ spox, 84$)$ and $P($ spoy, 84$)$ is observed.

The identified wavelet stationary periods $W h s(84, t)$ and $W h s(164, t)$ are transformed to a simplified model in Eq. 9, which produces a simplified deterministic TSI-HS data series from 1000 to 2100. The computed results in Table 2 show a close relation between the $P($ spox, 84$)$ maxima periods and minimum sunspots periods. The stationary model predicts minima in 1880 and 1960, which is seen in the TSI-HS reconstruction (Figure 3). The Eq. 9 model identifies three additional $P(h s c, t) \leq 0$ minimum periods, which are not shown in Table 2. These periods are (1296-1313), (1629-1656) and (1962-2002). The last period had a $P(h s c, t)=-0.40$ state in 1979 . The model estimates a minimum $P(h s c, t) \leq-0.5$ in the period (2030-2065), a grand minimum $P(h s c, t) \leq-1.0$ period (2044-2054) and a local minimum irradiation state in approximately 2050. 
These estimates support the identification of Uranus, in resonance with Neptune, as the major cause of TSI-HS variability.

The implication of this result is a chain of events between the solar inertial motion due to the large planets and the TSI-HS variability. The SPO period $P($ spoy, 84$)$, controlled by the 84-year Uranus period may serve as a reference for the TSI-HS variability.

\subsection{TSI-LS variability}

The TSI-LS variability is influenced by the periods from the large planets, as shown in Table 1. The major variability is, however, influenced by the long stationary periods $P(l s, 125)$ and $P(l s, 210)$. A stationary period is dependent on a stationary source. The autocorrelations in Figure 8 indicate a $3 / 2$ and $5 / 2$ combination resonance to $P(l s, 84)$, which produce the stationary periods $P(l s, 3 * 84 / 2=126)$ and $P(l s, 5 * 84 / 2=210)$. The same stationary deterministic periods produce a new set of subharmonic periods $P(l s, n * 126)$ and $P(l s, n * 210)$ for $n=1,2,3$. When $P(l s, 125)$ and $P(l s, 210)$ are related to the stationary Uranus period $P(U, 84.02)$, they will produce a set of subharmonic stationary periods. The period $P(l s, 3 * 84 / 2=126.03)$ will produce the subharmonic periods $P(l s, 2 * 126.03=252.06), P(l s, 3 * 126.03=378.09)$, $P(l s, 4 * 126.03=504.12), P(l s, 5 * 126.03=630.15)$ and $P(l s, 6 * 126.03=$ $756.18)$. The period $P(l s, 5 * 84 / 2=210.05)$ will produce the subharmonic periods $P(l s, 2 * 210.05=420.1)$ and $P(l s, 3 * 210.05=630.15)$, which indicate that $P(l s, 126.03)$ and $P(l s, 210.05)$ have a coincidence resonance in periods of approximately 630 years (Nayfeh \& Mook, 2004, Ghilea, 2014).

The TSI-LS data series is reconstructed by Velasco Hererra et al. (2015), who performed a wavelet analysis of their TSI-PMOD and TSI-ACCRIM reconstructions for the years 1000-2100 and discovered periods of $11 \pm 3,60 \pm 20,120 \pm 30$ and $240 \pm 40$ years. They interpret the 11-year period as the Schwabe cycle and the 60-year period as the Yoshimura-Gleissberg cycle, which is associated with solar barycentric motion. The 120 years period they associated with solar magnetic activity (Velasco Hererra, 2013), and the 240-year period was asso- 
ciated with barycentric motion as discovered by Jose (1965). They concluded that the negative phase of the 120-year period coincides with the grand minima, the positive phase of the 120-year period coincides with the grand maxima. The next minimum should appear between 2010 and 2070 according to this interpretation. Long periods were also identified by McCracken et al. (2014), who analyzed ${ }^{10} \mathrm{Be}$ from cosmic ray variation over the past 9,400 years by a Fourier spectrum analysis and identified three related period pairs (65 and 130), (75 and 150), and (104 and 208) in addition to periods 350, 510 and 708 years. The identified 210-year period is known as the de Vries/Suess period. It is identified as a stationary period of approximately 210 years in the radiocarbon dating of pine tree rings of the last 8,000 years (Suess, 1980) .

The identified periods in this investigation support the study by Suess (1980); 835 Velasco Hererra et al. (2015) and McCracken et al. (2014). The new information reveals that all long periods in the TSI-LS variability are traced to the deterministic Uranus period $P(U, 84.02)$. This study confirms that the TSI-LS variability is dominated by deterministic periods and explains why the identified periods from Suess (1980) and McCracken et al. (2014) are found in series of 8,000 and 9.400 years length.

An identification of stationary periods in TSI variability can provide information about future irradiation variability and expected long-term climate variation. The computed minima from the deterministic model (Eq. 13) show a close relation between the solar grand minimum periods and the computed minimum periods from the model (Table 2). From this deterministic model, we may expect a new TSI minimum $P(l s c, t) \leq-0.5$ for the period $2040-$ 2080, a Dalton state level $P(l s c, t) \leq-0.7$ in the time-period $2048-2068$ and a minimum state $P(l s c, t)=-0.9$ at approximately 2060 .

\subsection{Sunspot variability}

A study of the sunspot data series from 1610 identified the first dominant periods $P(s n, 11), P(s n, 55), P(s n, 110)$ and $P(s n, 210)$, as shown in Table 1 . The period $P(s n, 11)$ is a mean estimate from a time-variant phase. The wavelet 
spectrum in Figure 11 shows that the period $P(s n, 55)$ has a time variant amplitude that is controlled by the period $P(s n, 110)$. The stationary first period $P(s n, 11)$ is related to the Jupiter period $P(J, 11.862)$ and produces the subharmonic periods $P(s n, 5 * 11.862=59.31), P(s n, 2 * 59.31=118.62)$ and $P(s n, 4 * 59.31=237.24)$.

The periods have a combination resonance to the Uranus period $P(U, 84.02)$. The autocorrelation spectrum in Figure 12 shows that the period $P(s n, 55)$ has a $2 / 3$ combination resonance to the period $P(s n, 84)$. This finding indicates that the identified period $P(s n, 55)$ is a stationary period that is controlled by the Uranus period $P(U, 84.02)$ from the $2 / 3$ relation $2 * 84.02 / 3=56.01$ years. This first stationary period is expected to produce a new set of subharmonic periods of approximately $2 * 56=112$ and $4 * 56=224$ years. The identified period $P(s n, 210)$ has a $5 / 2$ combination resonance to the Uranus period $P(U, 84.02)$ by the relation $5 * 84.02 / 2=210.05$ years. Table 1 shows a close relation between the identified TSI period $P(h s, 11), P(l s, 11)$, the sunspots period $P(s n, 11)$ and the Jupiter period $P(J, 11.862)$. This study has demonstrated that the Uranus period $P(U, 84.02)$ introduces a deterministic TSI period of approximately $5 *$ $84 / 2=210.05$ years, a deterministic sunspots period of approximately $4(2 *$ $84.02 / 3)=224.05$ years and a mean coincidence period of 217 years.

The sunspot data series has been investigated for decades. Schwabe (1844) proposed Jupiter as a source for $P(s n, 11)$, and Ljungman (1879) presented the theory that the long-term herring biomass fluctuation was related to a 111year sunspot cycle. The 210-year de Vries/Suess period is related to a climate cycle (Suess, 1980). The new information from this study is that the 210year de Vries/Suess period, which is identified as a deterministic period in the TSI-LS and the sunspots data series, has its minimum at a phase difference of $0.35 \pi(\mathrm{rad} /$ year $)$ from the SPOx maximum in 1797, which coincide with Uranus 880 perihelion. This shows that they are controlled by the same 84-year Uranus period,

This study shows that solar variability and TSI variability have deterministic coincidence periods of approximately 11 and 210-220 years. The deterministic 
model of the solar variability indicates that we may expect a new sunspot solar phenomenological model proposed by McIntosh et al. (2014); McIntosh \& Leamon (2014), and McIntosh \& Leamon (2015) which explains the deep minima, 
requires a modulation of the solar differential rotation which may be a result of TSI and sunspot data series variability have stationary oscillating periods that 
is controlled by the gravity from the large planets Jupiter, Saturn, Uranus and Neptune. The identified periodic relation between the solar system oscillation and the TSI variability, indicates a chain of events between the solar system gravity force oscillation and the TSI variability. A possible chain of events is that the GO between the Sun and the large planets influences the solar dynamo oscillation, which produces the TSI variability and the sunspot variability.

The study demonstrates that the major TSI variability and sunspot variability are controlled by the 11-year Jupiter period and the 84-year Uranus period. The TSI data series from 1700 has a variability that is controlled by the 11-year Jupiter period and the 84-year Uranus period. The TSI data series from 1000 has a stationary dominant period of approximately 125 years, which is controlled by a $3 / 2$ resonance to the 84 -year Uranus period, and a 210 -year period 955 by a $5 / 2$ resonances to the 84 -year Uranus period. The stationary periods of approximately 125 and 210 years introduce a new set of deterministic subharmonic periods. The study confirms the deterministic relation between 210-year variability and TSI variability, which is known as the 210-year de Vries/Suess period (Suess, 1980).

The identified stationary periods in TSI variability and sunspot variability are transformed to deterministic models of TSI oscillation and sunspot oscillation. The close relation between the computed deterministic model minima and the known minimum periods since 1000 confirms the identified periods from this study. The deterministic model of sunspots and TSI computes a new Daltontype sunspot minimum from 2025 to 2050 and a new Dalton-period-type TSI minimum from approximately 2040 to 2065 .

\section{Acknowledgements}

We thank N. Scafetta and V. M. Velasco Hererra for providing the data sets for TSI we have used in this investigation. We also thank an anonymous referee for valuable suggestions, helping us to improve the manuscript. 


\section{References}

Abreu, J.A., Beer, J., Steinhilber, F., Tobias, S.M. and Weiss, N.O., 2008, For how long will the current grand maximum of solar activity persist?. Geophys. Res. Lett., 35, L20109

Blizard, J.B., 1981, Solar Motion and Solar Activity, Bull. Am. Astron. Soc, 13, 876

Charbonneu, P. 2010, Dynamo Model of the Solar Cycle, Living Rev. Sol. Phys.,7,3, www.livingreviews.org/lrsp-2010-3

Charvátová I and Hejda, P., 2014, Responses of the basic cycles of 178.7 and 2402 year in solar-terestrial phenomena during the Holocene, Pattern Recognit. Phys. 2, 21-26.

Cionco, R.G. and Soon, W., 2015, A phenomenological study of the timing of solar activity minima of the last millennium through a physical modeling of the Sun-Planets Interaction, New Astron., 34, 164-171.

Clette, F., Svalgaard, L., Vaquero, J. M, and Cliver, E.W., 2014, Revisiting the Sunspot Number, in The Solar Activity Cycle, V 53 Space Sciences Series of ISSSI pp 35-103.

Daubechies I. 1992. Ten lectures of wavelet. SIAM J. Math. Anal. 24:499-519.

de Jager, C. and Duhau, S., 2011, The Variable Solar Dynamo and the Forecast of Solar Activity; Influence on Terrestrial Surface Temperature, in Global warming in the 21st Century, J.M.Cossia (ed), ISBN978-1-61728-980-4. Nova Science Publishers, Inc.

Duhau S. and de Jager, C., 2008. The Solar Dynamo and Its Phase Transition during the Last Millennium. Sol. Phys., 250: 1-15.

Eddy, J.A., 1983, The Maunder Minimum A reappraisal, Sol. Phys., 89, 195207, DOI: 10007/BF00211962. 
Eddy, J.A., 1976, The Maunder Minimum, Science, 192,1189-1202

Fairbridge, R.W. and Shirley, J.H., 1987, Prolonged minima and the 179-yr cycle of the solar inertial motion, Sol. Phys., 110, 191-220.

Granpierre, A., 1996, On the origin of solar periodicity. Astrophys. Space Sci. 243,393-400.

Granpierre, A., 1990, How is the Sun working?, Sol. Phys., 128, 3-6.

Hassan, D. et al., 2016, Sunspots and ENSO relationship using Markov method, 
Hoyt, D.V. and Schatten, K.H., 1998a, Group Sunspot Numbers: A New Solar Activity Reconstruction, Sol. Phys., 179, 189-219.

Hoyt, D.V. and Schatten, K.H., 1998b, Group Sunspot Numbers: A New Solar Activity Reconstruction, Sol. Phys., 181, 491-512.

Hoyt, D. V., Schatten,K.H., and Nesme-Ribes, E., 1994, The one hundredth year of Rudolf Wolfs death: Do we have the correct reconstruction of solar activity?, Geophys. Res. Lett., 21, 2067-2070.

Hoyt, D. V., and Schatten K. H. 1993. A Discussion of Plausible Solar Irradiance Variations, 1700-1992. J. Geophys. Res., 98, 18895-18906.

Hung, C.-C. ,2007, Apparent relations between solar activity and solar tides caused by the planets, NASA/TM-2007-214817.Technical Memorandum.

Jose, P. D. 1965, Sun's motion and sunspots, Astron. J., 70, 193-200, 1965.

Ljungman, A. V., 1879. Bidrag till lösningen af frågan om de stora sillfiskenas sekulära periodisitet. Tidskrift for Fiskeri, 5: 257-268. (In Swedish).

Ljungquist, F.C., 2010, A new reconstrunction of temperature variability in the extra-tropical Northern hemisphere during the last two millennia, Geogra. Ann., 92 A(3) 339-351.

Matlab. 2015. Matlab. Wavelet Toolbox. Users Guide. The Math Works Inc. Maunder, E.W., 1890, Professor Spoerer's Researches on Sun-spots, MNRAS, $50,251-252$.

McCracken, K.G., Beer, J., and Steinhilber, F., 2014. Evidence for Planetary Forcing of the Cosmic Ray Intensity and Solar Activity Throughout the Past 9400 Years., Sol. Phys., 289,3207-3229, doi:10.1007/s11207-014-0510-1

McIntosh, S.W., Wang, X., Leamon, R.J. et al., 2014a, Deciphering Solar Magnetic Activity I. On the Relationship Between the Sunspot Cycle and the Evolution of Small Magnetic Features, ApJ, 792,12-30, doi:10.1088/0004637X/792/1/12 
McIntosh, S.W. and Leamon, R.J, 2015, Deciphering Solar Magnetic Activity:

On Gand Minima in Solar activity, Stellar and Solar Physics, vol 1, id.2, doi:10.3389/fspas.2015.00002

McIntosh, S.W. and Leamon, R. J., 2014b, On Magnetic Activity Band Overlap, Interaction, and the Formation of Complex Solar Active Regions, ApJ, 796, L19-24, doi:10.1088/2041-8205/796/1/L19

Mörner, N.-A., 2010, Solar Minima, Earth's rotation and Little Ice Ages in the past and in the future The North Atlantic-European case, Global Planet. Change, 72, 282-293, doi:10.1016/j.gloplacha.2010.01.004.

Mörner, N.-A., Scafetta, N. \& Solheim, J.-E., 2015, The January 7 giant solar flare, The simultaneous triple planetary conjunction and additional records at Troms $\varnothing$, Norway, In: Planetary infuence on the Sun and the Earth, and a modern book-burning, Ed: N.-A. Mörner, Nova publ, ISBN 978-1-63482-8376, p.33-38.

Nayfeh A. H. and Mook D. T., 2004. Nonlinear Oscillations. WILEY-VCH Verlag GmbH \& Co. KGaA, Weinheim. ISBN-10:0-471-12142-8. period cycles of the Sun's activity recorded in direct solar data and proxies, Sol. Phys., 211,371-394.

Peristykh, A. N. and Damon, P. E., 2003, Persistence of the Gleissberg 88year solar cycle over the last $\sim 12,000$ years: Evidence from cosmogenic isotopes, J. Geophys. Res. Space Phys., 108,A1,pp.SSH 1-1, CiteID 1003, doi: 10.1029/2002JA009390

Scafetta, N., 2014. Discussion of the spectral coherence between planetary, solar and climate oscillations: a reply to some critiques, Astrophys. Space Sci, 354(2), 275-299, doi: 10.1007/s10509-014-2111-8 
Schwabe, H., 1844, Sonnen-Beobactungen im Jahre 1843, Astron. Nachr. 21, (495), 233-236.

Sharp, G. J., 2013, Are Uranus \& Neptune Responsible for Solar Grand Minima and Solar Cycle Modulation?, Int. J. Ast. \& Astrophys., 3, 260-273, dx.doi.org/10.4236/ijaa.2013.33031.

Shepherd, S. J., Zharkov, S. I. and Zharkova, V. V. 2014, Prediction of Solar Activity from Solar Background Magnetic Field Variations in Cycles 21-23. ApJ. 795(1), article id. 46,8 pp, doi: 10.1088/0004-637X/795/1/46

Solanki, S. K., Usoskin, I. G., Kromer, B., Schüssler, M. and Beer, J.. 2004, Unusual activity of the Sun during recent decades compared to the previous 11,000 years, Nature, 431, 1084-1087.

Soon, W, Connolly, R. and Connolly, M., 2015, Re-evalutating the role of solar variability on Northern Hemisphere temperature trends since the 19th century, Earth-Sci. Rev., 150, 409.

1095

Suess, H. E., 1980, The Radiocarbon Record in Tree Rings of the Last 8000 Years, Proc. of the 10th international Conference on Radiocarbon Dating, Bern and Heidelberg 1979, Radiocarbon, 22, 200-209.

Usoskin, I.G., et al. 2014, Evidence for distinct modes of solar activity, A\& A, 562, L10, DOI: 10.1051/0004-6361/201423391.

1100 Usoskin, I. G., 2013, A History of Solar Activity over Millennia, Living Rev. Solar Phys., 10,1. URL(accessed 2014, 0530): http://www.livingreviews.org/lrsp-2013-1 
Usoskin, I. G., Solanki, S. K. and Kovaltsov, G. A., 2007, Grand minima and maxima of solar activity: new observational constraints A\&A, 471, 301-309.

Usoskin, I. G., Solanki, S. K., Schüssler, M., Mursula, K. and Alanko, K., 2003,, Millennium-Scale Sunspot Number Reconstruction: Evidence for an Unusually Active Sun since the 1940s, Phys. Rev. Lett., 91, 211101.

Velasco Herrera, V. M, Mendoza, B. and Velasco Herrera, G., 2015, Reconstruction and prediction of the total solar irradiance: From the Medival Warm Period to the 21st century, New Astron., 34, 221-233.

Velasco Herrera,V. M., 2013, Homage to the Discovery of Cosmic Rays, the Meson-Muon and Solar Cosmic Rays, Nova publ., Jorge A., Perez-Peraza (Eds), chap. 15, 469.

Velasco, V. M. and Mendoza, B., 2008, Assessing the relationship between solar activity and some large scale climatic phenomena, Adv. Space Res., 42, 866878, DOI: $10.1016 /$ j.asr.2007.05.050

Willson, R.C., 2014, ACRIM3 and the Total Solar Irradiance database, Astrophys. Space Sci, 352(2), 341-352, doi: 10.1007/s 10509-014-1961-4

Wilson, I. R. G., 2013, The Venus-Earth-Jupiter spin-orbit coupling model, Pattern Recognit. Phys.,1,147-158, doi:10.5194/prp-1-147-2013-

Wolf, C.L. and O'Donovan, A. E., 2007, Coupled groups of g-modes in a Sun with a mixed core, ApJ. 661, 568-585.

Wolf, R.,1859, Extract of a Letter to Mr. Carrington, MNRAS,19,85-86.

Wolf, C.I. and Patrone P. N., A New Way that Planets Can Affect the Sun, 1125 2010, Sol. Phys, 266, 227-246, DOI 10.1007/s11207-0101-9628-y.

Wolf, R.,1861, Abstract of his latest Results, MNRAS, 21, 77-78

Zhao, J., Bogart, R. S., Kosovichev, A. G., Duvall, T. L. Jr., and Hartlep, T., 2013, Detection of Equatorward Meridional Flow and Evidence of Double-cell Meridional Circulation inside the Sun, ApJ, 774, L29. 
Zharkova, V. V., Shepherd, S. J., Popova, E. and Zharkov, S. I.,2015, Heartbeat of the Sun from Principal Component Analysis and prediction of solar activity on a millennium timescale, www.nature.com/Scientific reports, 5:15689, DOI: $10.1038 /$ srep1569.

Table 1: Identified stationary periods

\begin{tabular}{|l|c|c|c|c|c|c|c|c|c}
\hline Data & Per,R & Per,R & Per,R & Per, R & Per, R & Per, R & Per, R & Per, R & Per,R \\
\hline Planet & $P J$ & $P S$ & $P(55)$ & $P U$ & $P(110)$ & $P(125)$ & $P N$ & $P(210)$ & $P(373)$ \\
period (yr) & 11.862 & 29.447 & $2 P U / 3$ & 84.02 & $4 P U / 3$ & $3 P U / 2$ & 164.79 & $5 P U / 2$ & $9 P U / 2$ \\
\hline P(SPO) & $s p o y, 12$ & $s p o y, 29$ & & $s p o y, 84$ & & & $s p o y, 165$ & & \\
R= & 0.98 & 0.95 & & 0.90 & & & 0.90 & & \\
\hline TSI-HS & $h s, 11$ & & & $h s, 84$ & & & $h s, 164$ & & \\
R= & 0.55 & & & 0.65 & & & 0.70 & & \\
\hline TSI-LS & $l s, 11$ & $l s, 29$ & & $l s, 83$ & & $l s, 125$ & & $l s, 210$ & $l s, 373$ \\
R= & 0.80 & 0.20 & & 0.17 & & 0.60 & & 0.35 & 0.50 \\
\hline SN & $s n, 11$ & & $s n, 55$ & $s n, 86$ & $s n, 110$ & & & $s n, 210$ & \\
R= & 0.73 & & 0.43 & 0.35 & 0.40 & & & 0.36 & \\
\hline
\end{tabular}


Table 2: Minimum periods from deterministic models

\begin{tabular}{|l|c|c|c|c|c|c|}
\hline Data series & Oort & Wolf & Spörer & Maunder & Dalton & Next \\
Minima periods: & $1010-1070$ & $1270-1340$ & $1390-1550$ & $1640-1720$ & $1790-1820$ & \\
\hline$P($ spoc, 84$) \max$ & 1043 & 1296 & 1462 & 1630 & 1797 & 2049 \\
$P($ spocy, 84$) \max$ & 1068 & 1326 & 1487 & 1654 & 1820 & 2073 \\
\hline HS model period & $1033-1055$ & $1369-1389$ & $1537-1554$ & $1706-1721$ & $1796-1830$ & $2035-2065$ \\
$P(h s c, t)$ & $<-1.0$ & $<-1.0$ & $<-1.0$ & $<-1.0$ & $<0$ & $<-0.7$ \\
\hline HS model min & 1040 & 1337 & 1547 & 1714 & 1810 & 2049 \\
$P(h s c, t)$ min & -1.30 & -1.23 & -1.87 & -1.13 & -0.33 & -1.0 \\
\hline LS model period & $1014-1056$ & $1276-1301$ & $1404-1435$ & $1657-1689$ & $1785-1810$ & $2045-2070$ \\
$P(l s c, t)$ & $<-1.0$ & $<-0.5$ & $<-1.0$ & $<-0.7$ & $<-0.7$ & $<-0.7$ \\
\hline LS model min & 1035 & 1289 & 1418 & 1672 & 1796 & 2060 \\
$P(l s c, t)$ min & -1.40 & -0.62 & -1.20 & -0.91 & -0.81 & -0.79 \\
\hline SN mode l period & $1019-1032$ & $1242-1256$ & $1467-1478$ & $1693-1699$ & $1802-1820$ & $2025-2050$ \\
$P($ snc,$t)$ & $<1.0$ & $<-1.0$ & $<-1.0$ & $<-1.0$ & $<-0.5$ & $<-0.5$ \\
\hline SN model min & 1026 & 1249 & 1473 & 1696 & 1811 & 2035 \\
$P($ snc, $t)$ min & -1.7 & -1.18 & -1.13 & -1.04 & -0.79 & -0.84 \\
\hline
\end{tabular}




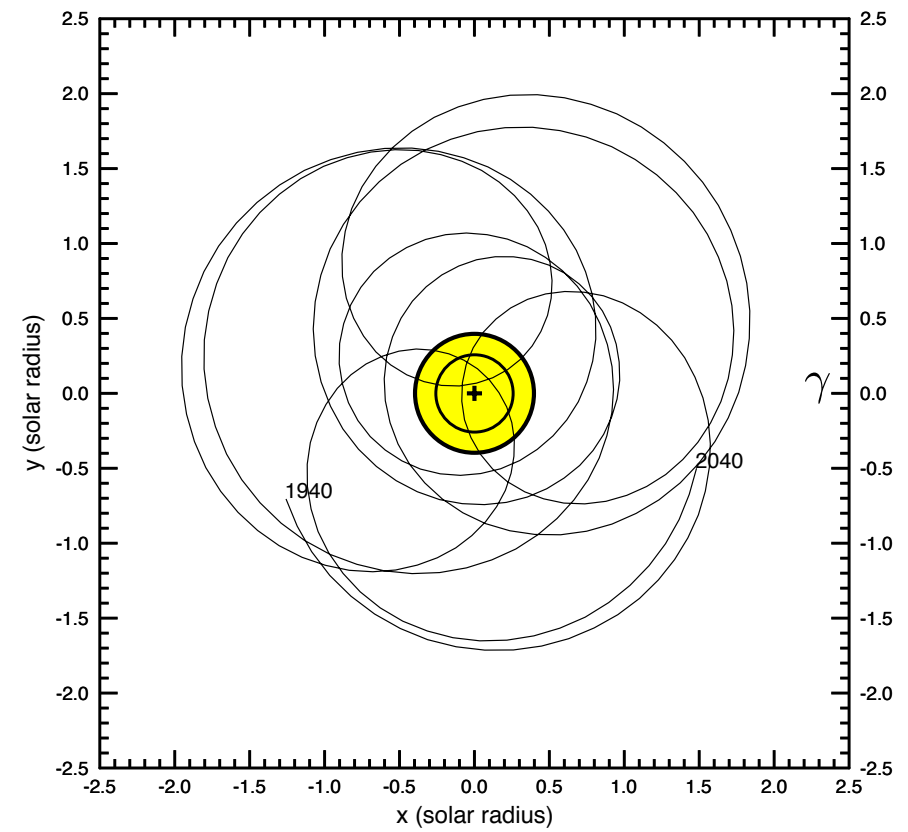

Figure 1: Orbit of the solar center with respect to the solar system barycenter (SSB) (+) for the period 1940-2040 in the ecliptic plane defined in the direction of the Earth's vernal equinox $(\Upsilon)$. The outer yellow circle represents the diameter of the Sun, and the inner circle at radius $0.65 r_{s}$ represents a shell where the potential energy $(\mathrm{PE})$ of the solar radiative zone can be affected if the solar center moves closer to the SSB (Cionco \& Soon, 2015). 


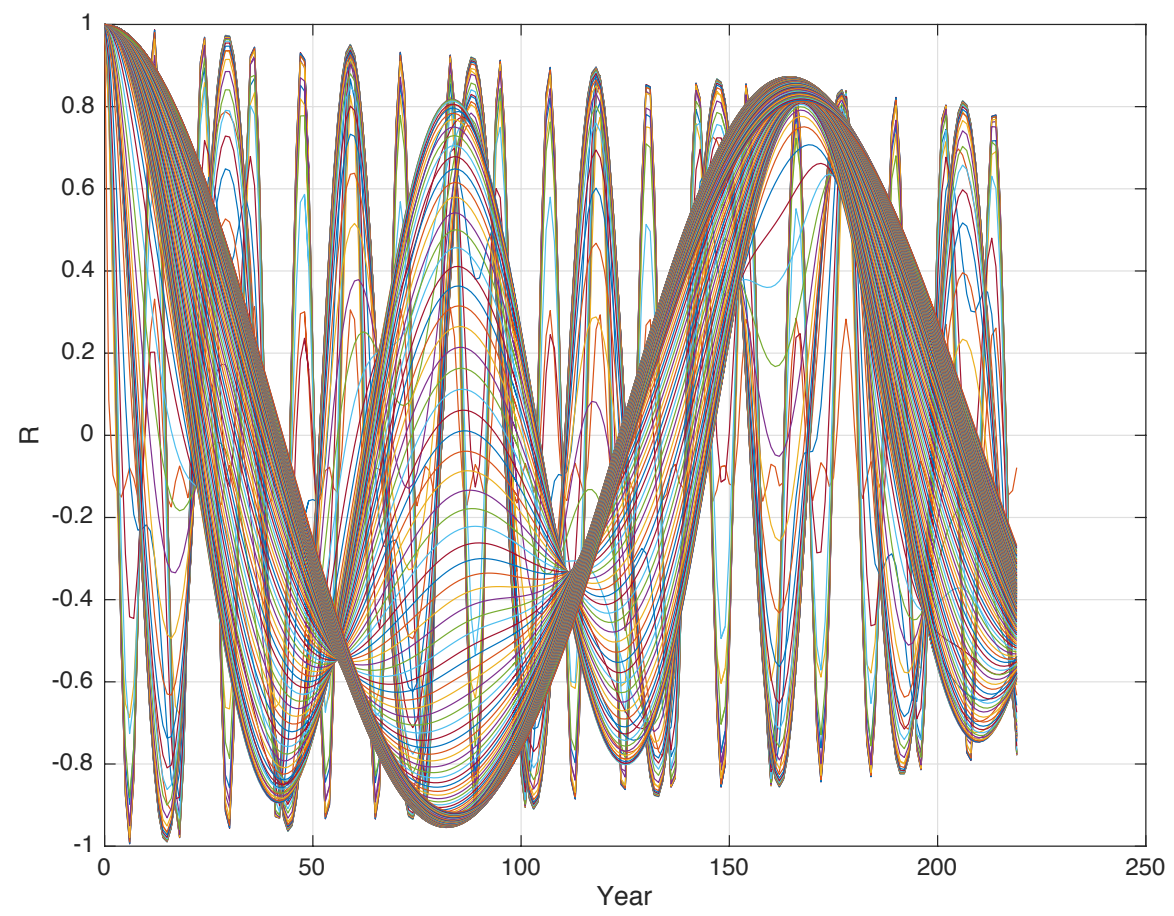

Figure 2: Autocorrelations Rspoy $(s, m)$ of Sun $_{45}$ position oscillation (SPOy) wavelet spectrum $W \operatorname{spoy}(s, t)$ for $m=0 \ldots 225$. Each colored line represents a single autocorrelation function. 


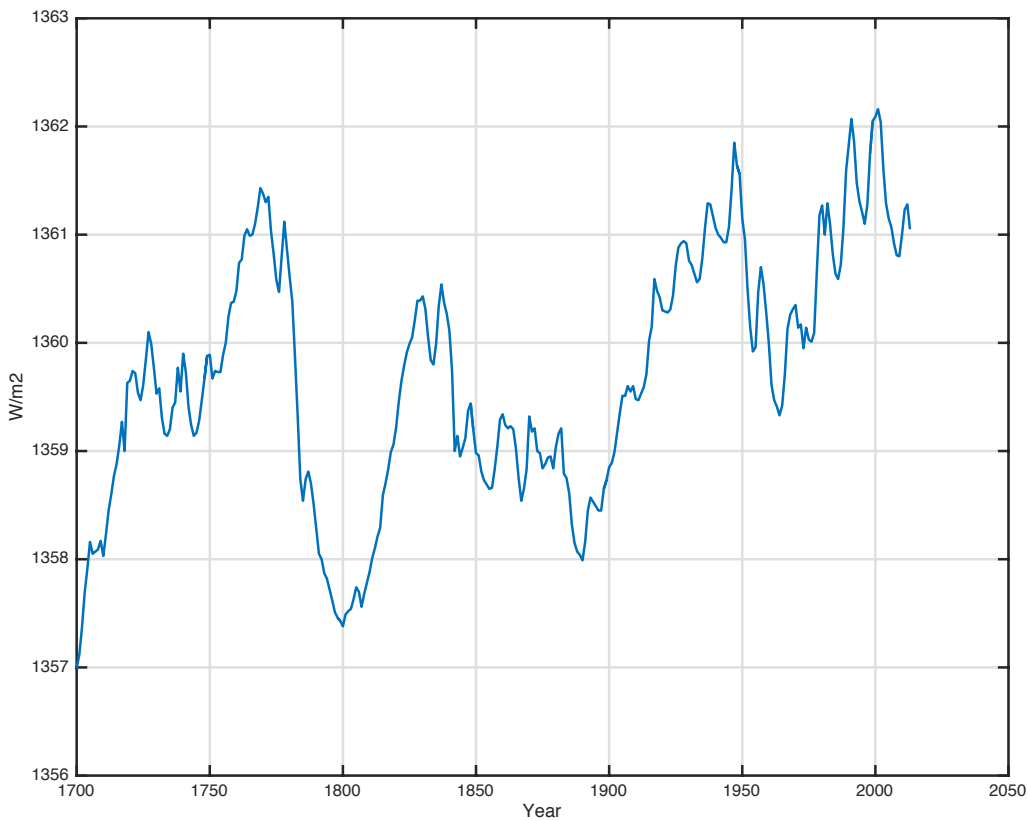

Figure 3: TSI-HS total solar irradiance from A.D. 1700 to 2013 (Scafetta \& Willson 2014). 


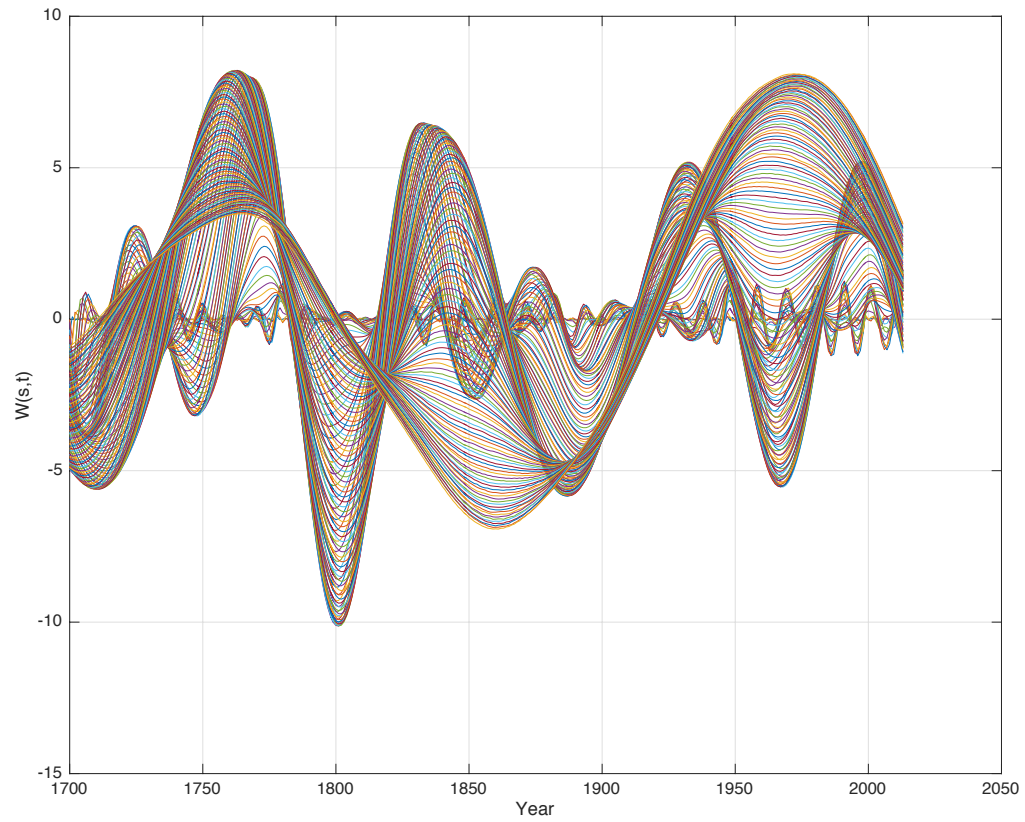

Figure 4: Wavelet spectrum $W h s(s, t)$ of the TSI-HS data series, for $s=1 \ldots 190$. 


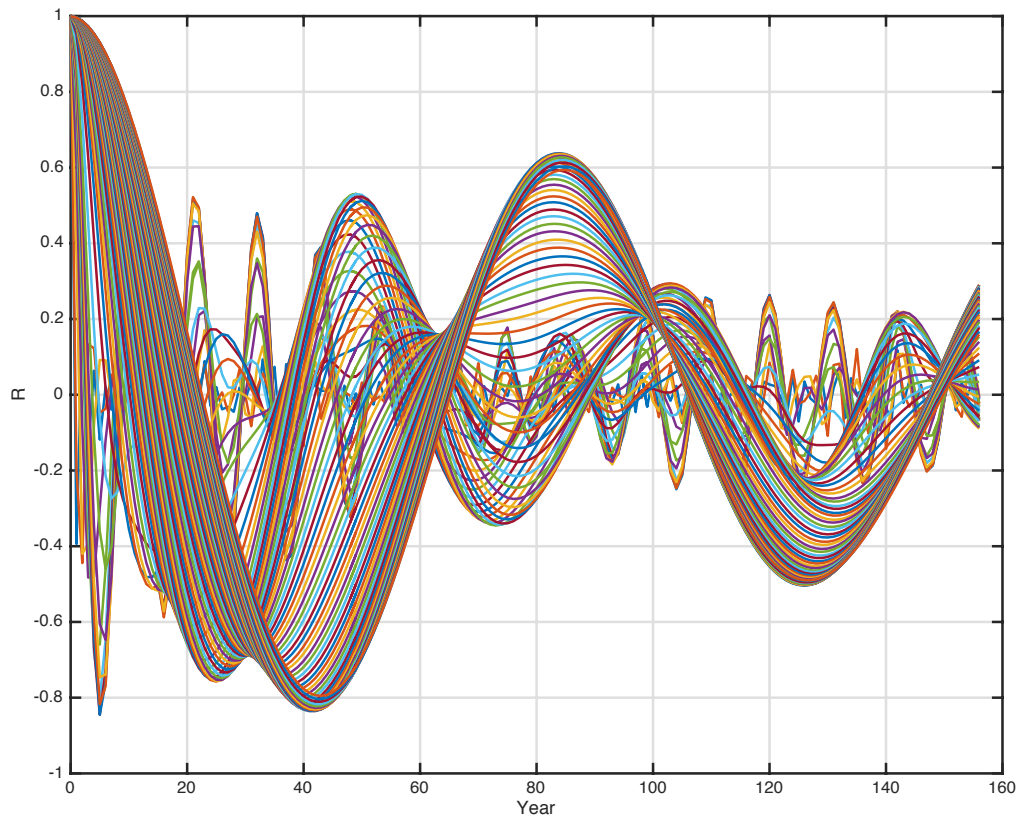

Figure 5: Autocorrelations $R h s(s, m)$ of the TSI-HS wavelet spectrum $W h s(s, t)$ for $s=$ $1 \ldots 190$ and $m=1 \ldots 160$. 


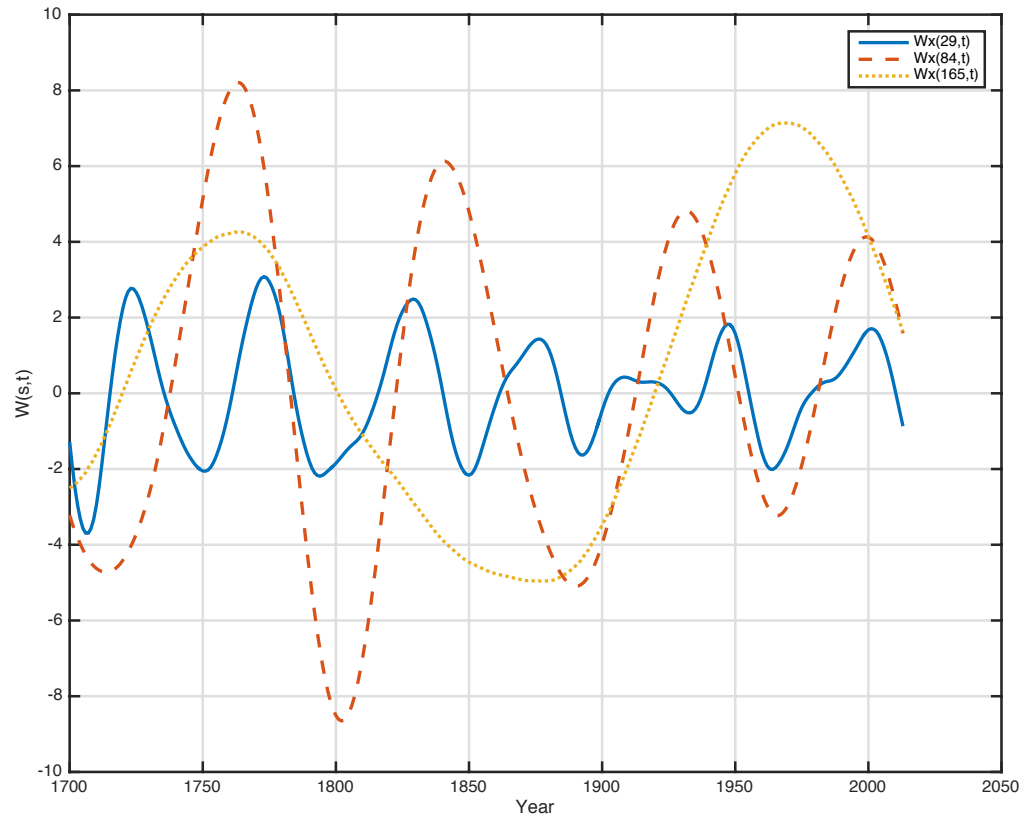

Figure 6: The identified stationary wavelet periods $W h s(49, t), W h s(86, t)$ and $W h s(165, t)$ from the TSI-HS wavelet spectrum $W h s(s, t)$. 


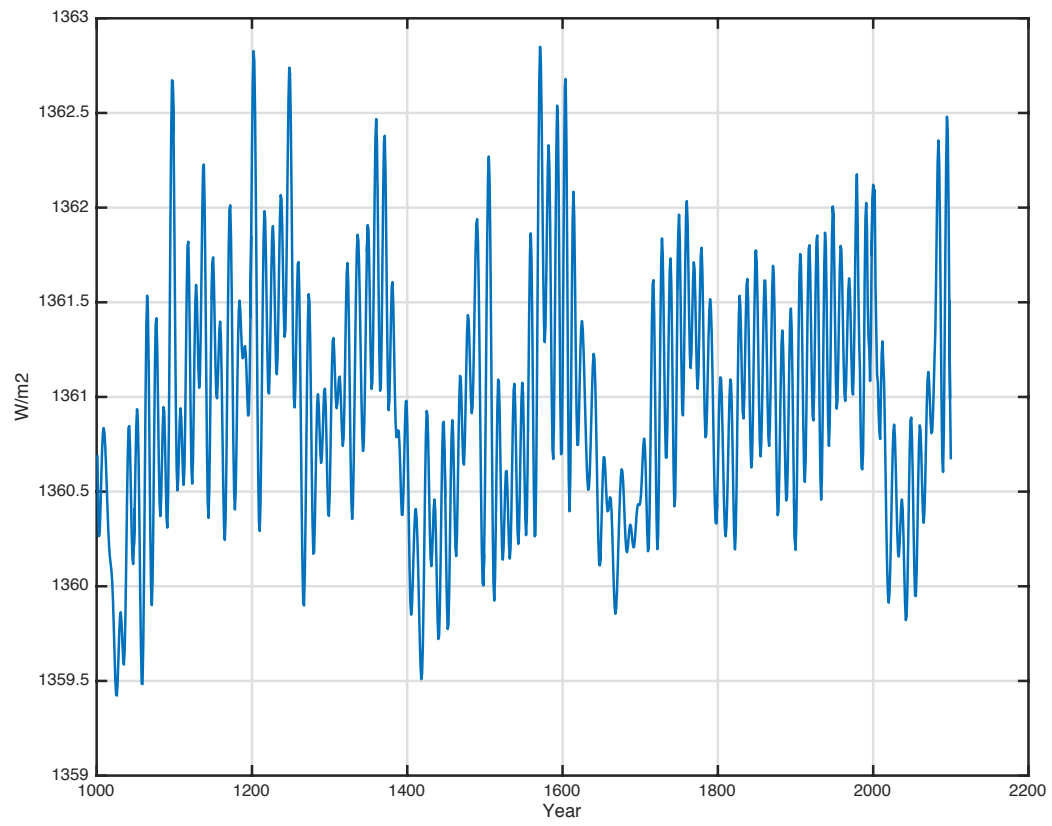

Figure 7: TSI-LS data series from 1000 to 2100 Velasco Hererra et al. (2015). 


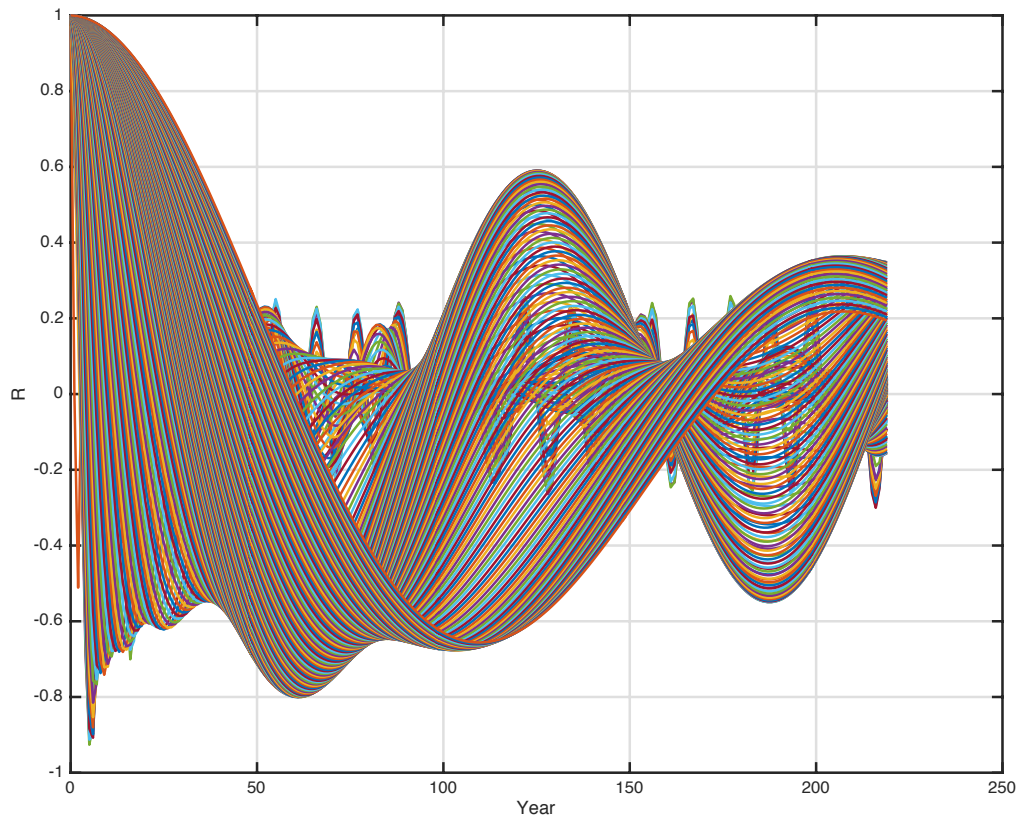

Figure 8: Autocorrelations $R l s(s, m)$ of the TSI-LS wavelet spectrum $W l s(s, t)$ for $s=$ $0 \ldots 600$, and $m=0 \ldots 225$. 


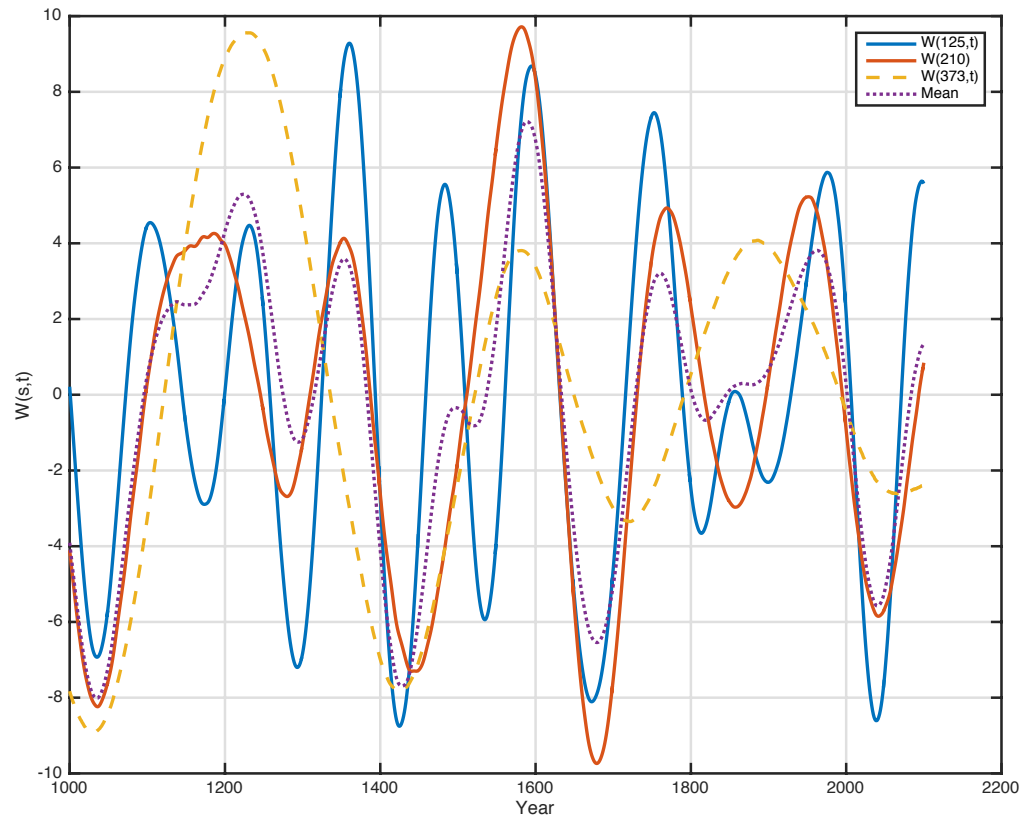

Figure 9: Identified long stationary wavelet periods $W l s(125, t), W l s(210, t) W l s(373, t)$ and their mean value 


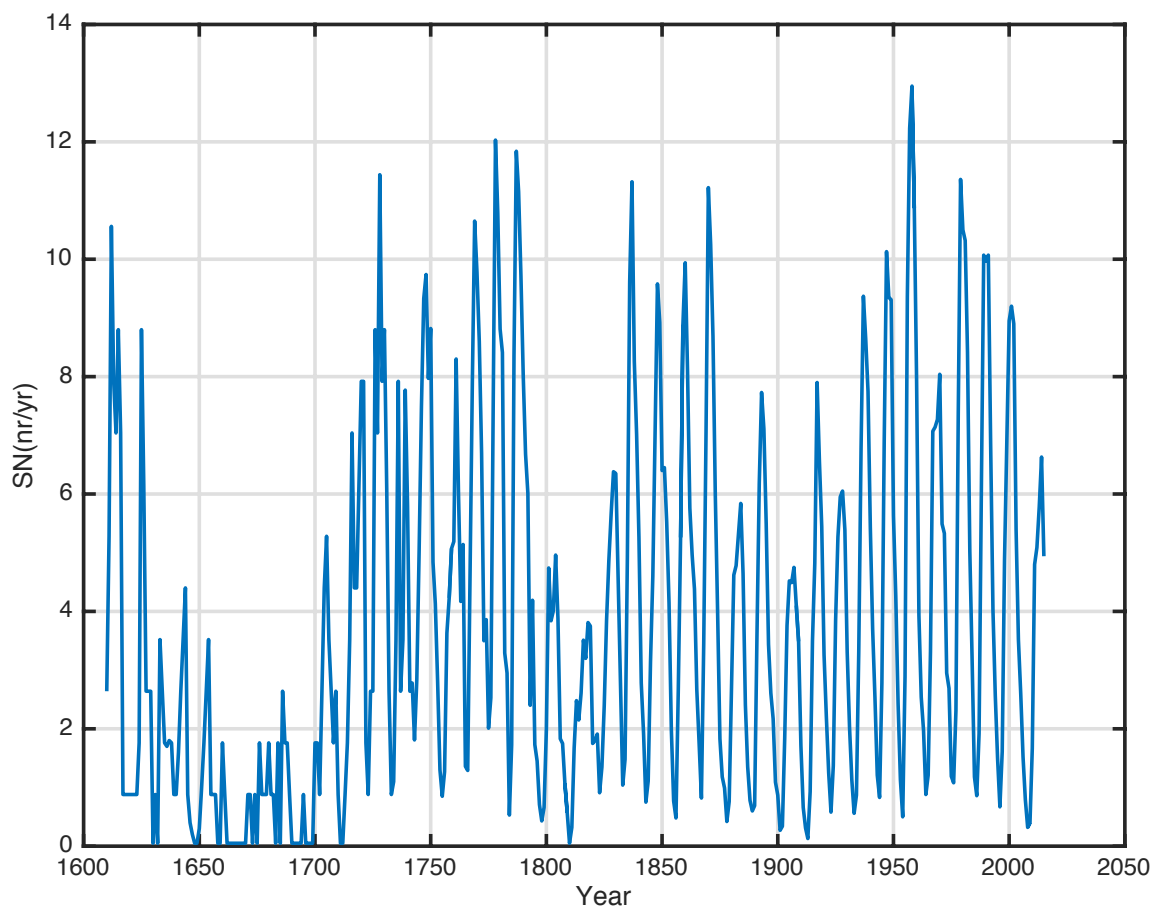

Figure 10: Solar variability represented by the yearly average group sunspot number series $S N(t)$, estimated from A.D.1610 to 2015 (SILSO data/image, Royal Observatory of Belgium, Brussels) 


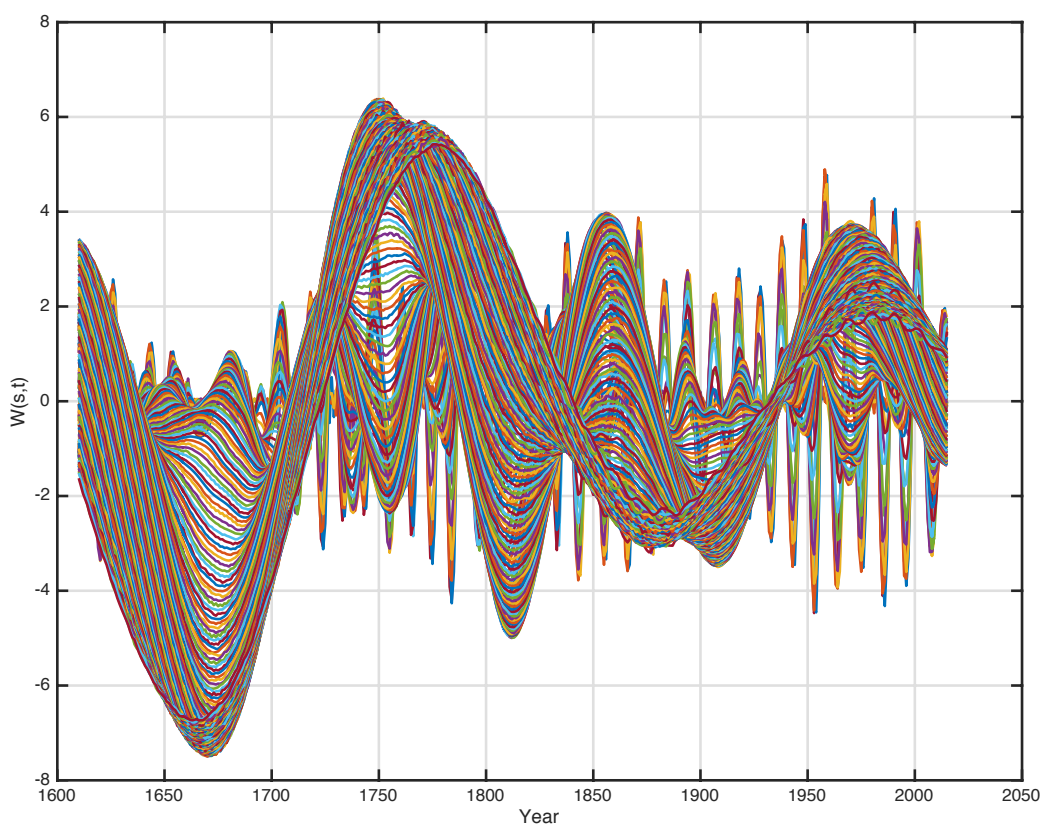

Figure 11: Wavelet spectrum $W s n(s, t)$ of the sunspot data series $S N(t)$. 


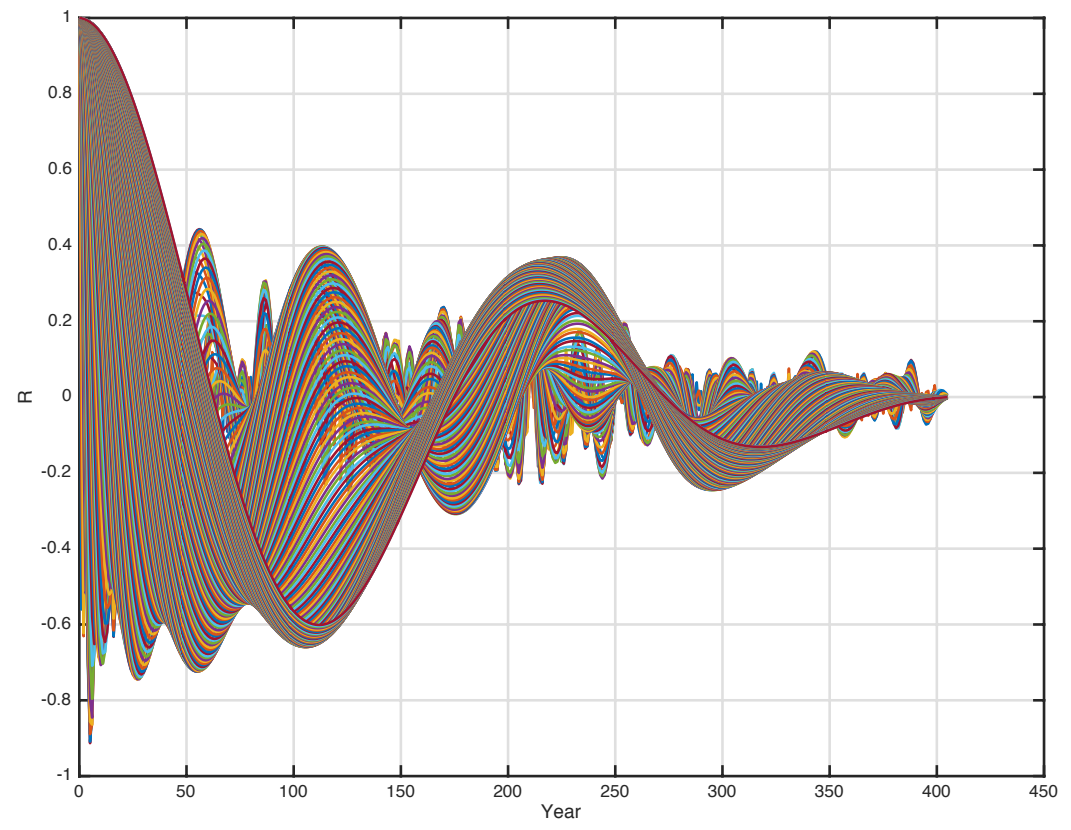

Figure 12: Autocorrelations $R s n(s, m)$ of the $\mathrm{SN}$ wavelet spectrum $W s n(s, t)$ for $s=0 \ldots 240$, and $m=0 \ldots 400$. 